\title{
Groundwater Maps of the Hanford Site, June 1994
}

\author{
J. A. Serkowski \\ W. A. Jordan \\ M. J. Hartman \\ Date Published \\ December 1994
}

Prepared for the U.S. Department of Energy Office of Environmental Restoration and Waste Management
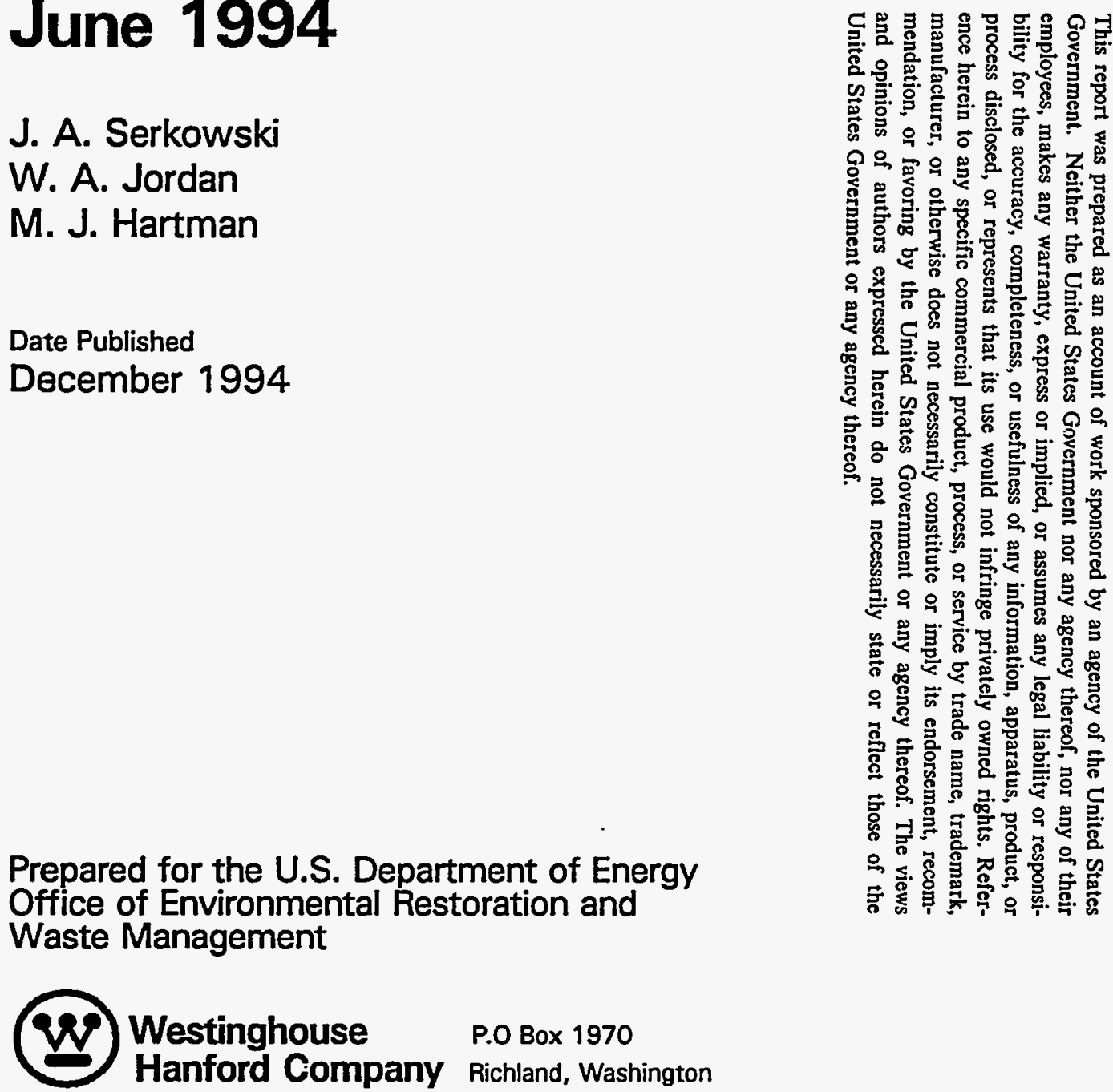

Hanford Operations and Engineering Contractor for the

U.S. Department of Energy under Contract DE-AC06-87RL10930 


\section{DISCLAIMER}

Portions of this document may be illegible in electronic image products. Images are produced from the best available original document. 


\section{RELEASE AUTHORIZATION}

Document Number: WHC-EP-0364-9

Document Title: Groundwater Maps of the Hanford Site, June 1994

Release Date: $\quad 12 / 29 / 94$

This document was reviewed following the procedures described in WHC-CM-3-4 and is:

APPROVED FOR PUBLIC RELEASE

WHC information Release Administration Specialist:

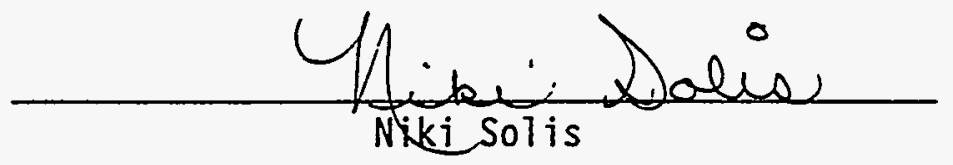


WHC-EP-0394-9

\section{CONTENTS}

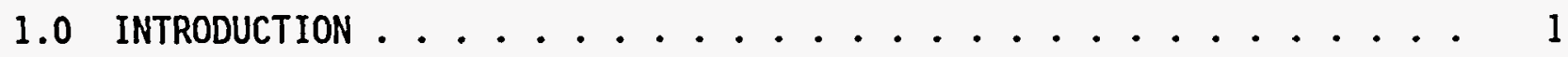

2.0 DATA COLLECTION, MANAGEMENT, EVALUATION, AND PRESENTATION . . . 1

3.0 HANFORD SITE MAPS ................... 3

3.1100 AREAS MAPS .................... 3

3.2200 AREAS MAPS ........................ 3

$3.3300 / 1100$ AREAS MAPS ................. 4

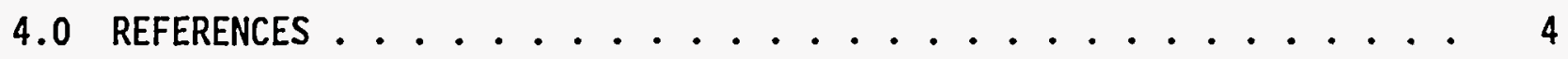
APPENDIX

A. Water Leve 1 Measurement Data .............. A-1

\section{LIST OF FIGURES}

1. Hanford Site Location Map. . . . . . . . . . . . . 7

2. Hanford Site Water Table ............... 9

3. 100 Area Location Map ................. 11

4. 100 Area Hater Table ............... . 13

5. 200 Area Location Map . . . . . . . . . . . . . 15

6. 200 Area Water Table . . . . . . . . . . . . 17

7. 300 Area Location Map . . . . . . . . . . . . . 19

8. 300 Area Water Table .............. 21 
WHC-EP-0394-9

This page intentionally left blank. 
WHC-EP-0394-9

GROUNDWATER MAPS OF THE HANFORD SITE

JUNE 1994

\subsection{INTRODUCTION}

This report is a continuation of reports (Kasza et al., 1994) that document the configuration of the uppermost unconfined aquifer beneath the Hanford Site (Figure 1). This series presents the results of the semiannual water level measurement program and the water table maps generated from these measurements. The reports document the changes in the groundwater level at the Hanford Site during the transition from nuclear material production to environmental restoration and remediation. In addition, these reports provide water level data to support the various site characterization and groundwater monitoring programs currently in progress on the Hanford Site. The reports are prepared for the U.S. Department of Energy, Office of Environmental Restoration and Waste Management, by the Hanford Site Operations and Engineering Contractor, Hestinghouse Hanford Company (WHC). This report fulfills reporting requirements specified in WHC-CM-7-5, Section 8.0, "Water Quality" (WHC 1993a), and described in the Environmental Monitoring Plan for the Hanford Site (DOE-RL 1991).

This report highlights the three major operations areas (the 100 , 200 , and 300/1100 Areas) where wastes were discharged to the soil. Each area includes a summary discussion of the data, a well index map, and a contoured map of the water table surface. Appendix A contains all of the data collected for this program, including data which were not used in the preparation of the water table maps.

The National Geodetic Vertical Datum of 1929 (NGVD29) is used as the vertical datum and Washington State Lambert coordinates are used for the horizontal 7ocation of the wells. Elevation data were converted from feet to meters for this report.

\subsection{DATA COLLECTION, MANAGEMENT, EVALUATION, AND PRESENTATION}

During June 1994, the depth to groundwater was measured in 814 wells on the Hanford Site by personnel from the Well Services Group under the direction of the Groundwater Management Group project leader. The procedure for measuring the depth to water and recording the data is contained in WHC-CM-7-7, Environmental Investigation and Site Characterization Manual, EII 10.2 (WHC 1993b). Field data were collected using the Groundwater Monitoring System, which employs a barcode interface to enter readings that have been taken manually with a steel tape from the reference point at the top of the well casing. The barcode hand-held computer downloaded the field data to a supervisory workstation that stores all groundwater level measurements. 
These data were then transferred to the Hanford Environmental Information System (HEIS) database and were later downloaded to a Paradox application program called GeoDAT to help organize, evaluate, and tabulate the data.

Data evaluation began with a review of the well construction and the quality of data obtained in June 1994. The following parameters were examined for most wells to determine the suitability of including the data in the water table surface model.

- Well location survey - A number of wells were rejected due to nonexistent or unreliable surveys.

- Screened interval - Hells were usually rejected if the screened interval exceeds $15 \mathrm{~m}$ in the water table (wells with long screened intervals were used where no alternate wells existed and vertical gradients were not considered significant); wells were also rejected if the screened interval is entirely below the top of the water table or the screened interval is not reliably known.

- Aquifer monitored - Only wells known to monitor the uppermost unconfined aquifer were included.

- Anomalous data - Data collected in June 1994 which does not agree with historic trends at that well were rejected.

Information for conducting this review was obtained from the HEIS database, personal communications with project scientists, and the most recent Resource Conservation and Recovery Act of 1976 (RCRA) Quarterly report (DOE-RL 1994). The notes column in the Appendix A data table reflects the results of this review.

Of the 814 wells measured in June 1994, 534 were selected to create a water table surface model. A model grid was generated using a kriging algorithm available in the $A R C /$ INFO $^{2}$ geographic information system (GIS) software. The GIS then created contour lines of equal water table elevations from the model grid. Contours are not present in areas where the basalt surface is believed to be above the water table, based on Connelly et al. (1992a and 1992b). Contours are dashed where the water table is poorly del ineated due to inadequate well coverage.

Hydrologists familiar with regional and local groundwater properties reviewed the maps to evaluate data interpolations made by the computer model. The contours were adjusted manually to reflect the known hydrologic environment.

\footnotetext{
'Paradox is a trademark of Borland International, Scotts Valley, California 95067-0001

${ }^{2}$ ARC/INFO is a trademark of Environmental Systems Research Institute, Inc., Redlands, Cal ifornia 92373-8100
} 


\subsection{HANFORD SITE MAPS}

Chapter 3.0 summarizes the results of the water table surface model generated from the June 1994 data. Figure 1 shows the relative locations of the detail facility area maps included in this document. To provide context, Figure 2 shows the water table surface for the entire Hanford Site. The detail maps are enlargements of this same sitewide model.

\subsection{AREAS MAPS}

For the purposes of this report, the 100 Areas comprise the various 100 Area reactor facilities and the surrounding land south of the Columbia River and north of Gable Mountain and Gable Butte. Reactor operations have ceased in all of the facilities, and environmental restoration activities are in progress. Maps for this area include Figure 3, Index Map of the 100 Areas Groundwater Monitoring Wells, and Figure 4,100 Areas Water Table. Some of the wells used have screened intervals exceeding $15 \mathrm{~m}$ within the top of the unconfined aquifer since the vertical gradients are not believed to be significant in this area.

Throughout most of the map area, groundwater flows from the unconfined aquifer into the Columbia River. West of the 100-B/C Area, water is believed to flow from the Columbia River into the aquifer.

The high water levels north of Gable Mountain are consistent with past measurements. Drillers' logs indicate the presence of fine-grained sediments in this area. The high water levels may be remnants of artificial recharge from pre-Hanford Site irrigation or may represent perched groundwater. The steep groundwater gradient in the southeastern portion of the map area is believed to result from the influence of the fine-grained sediments.

\subsection{AREAS MAPS}

These maps encompass the 200 East and 200 West Areas and the surrounding vicinity on the Hanford Site that was once referred to as the Separations Area. There are several active and many inactive liquid waste disposal facilities in this region. The 200 Areas maps consist of Figure 5, 200 Areas Monitoring Well Index Map, and Figure 6, 200 Areas Water Table.

Three facilities are notable in their impact on the water levels in this area: U Pond (216-U-10), located in the southwestern corner of 200 West Area, Z Plant (234-5), north of the U Pond Site, and B Pond (216-B-3), located east of 200 East Area. U Pond was deactivated in 1984 after 40 years of use as a disposal site for large volumes of liquid wastes. The relatively low hydraulic conductivity found beneath 200 West Area coupled with continued effluent discharge in cribs associated with Z P1ant has helped to maintain a significant water table mound under 200 West Area. Since 1984, the high point of the groundwater mound has shifted northward from $U$ pond, toward Z PIant. A steep gradient occurs east of 200 West Area as the water table intersects the higher conductivity sediments beneath 200 East Area. With higher conductivities, the water table beneath the 200 East Area is generally flat. 
B Pond, which continues to receive significant volumes of liquid effluent, does maintain a groundwater mound, influencing groundwater travel direction over a wide area.

\section{$3.3 \quad 300 / 1100$ AREAS MAPS}

These maps show the June 1994 water table measurements for the 300 Area and the adjacent 1100 Area. Liquid waste disposal continues only at the 300 Area Process Trenches, though clean river water is discharged during the summer months at the City of Richland well field recharge ponds located east of the 1100 Area. The 300/1100 Area maps consist of Figure 7, 300 Area Monitoring Wel1 Index Map, and Figure 8, 300 Areas Water Table.

The unconfined aquifer within this area is contained in the Ringold Formation and the Hanford formation (Swanson et a7., 1992). The top of the unconfined aquifer is close to the Hanford/Ringold contact. Generally, west of an imaginary north-south line near the west boundary of the 300 Area the unconfined aquifer is within the Ringold Formation. East of the line it is within the Hanford formation, which is more periable than the Ringold and may be expected to permit higher flow velocities.

The major influences on water table elevations in the map area are river fluctuations, irrigation, and river water recharged into the City of Richland well field near the 1100 Area. Water table elevation may also be dependent on recharge from the Yakima River to the west. Combined with high permiabilities in the Ringold formation, these influences result in a complex flow regime where local flow directions are difficult to predict.

\subsection{REFERENCES}

Connelly, M. P., B. H. Ford, and J. V. Borghese, 1992a, Hydrogeologic Model for the 200 West Groundwater Aggregate Area, WHC-SD-EN-TI-014, Westinghouse Hanford Company, Richland, Washington.

Connel7y, M. P., B. H. Ford, J. W. Lindberg, S. J. Trent, C. D. Delaney, and J. V. Borghese, 1992b, Hydrogeologic Mode7 for the 200 East Groundwater Aggregate Area, WHC-SD-EN-TI-019, Hestinghouse Hanford Company, Richland, Washington

DOE-RL, 1991, Environmental Monitoring Plan, DOE/RL 91-50, U.S. Department of Energy, Richland Field Office, Richland, Washington.

DOE-RL, 1994, Quarter7y Report for RCRA Groundwater Monitoring Data for Period Apri7 I through June 30, 1994, DOE/RL 94-36-2, U.S. Department of Energy, Richland Field Office, Richland, Washington.

Kasza, G. L., M. J. Hartman, H. A. Jordan, and J. V. Borghese, 1994, Ground Water Haps of the Hanford Site--December 1993, WHC-EP-0394-8, Westinghouse Hanford Company, Richland, Washington. 


\section{WHC-EP-0394-9}

Swanson, L. C., G. G. Kelty, K. A. Lindsey, K. R. Simpson, R. K. Price, and S. D. Consort, 1992, Phase I Hydrogeologic Summary of the 300-FF-5 Operab7e Unit, 300 Area, WHC-SD-EN-TI-052, Westinghouse Hanford Company, Richland, Washington.

WHC, 1993a, Environmental Comp7iance, HHC-CM-7-5, Westinghouse Hanford Company, Richland, Hashington.

WHC, 1993b, Environmental Investigations and Site Characterization Manual, WHC-CM-7-7, Westinghouse Hanford Company, Richland, Washington. 
WHC-EP-0394-9:

This page intentionally left blank. 


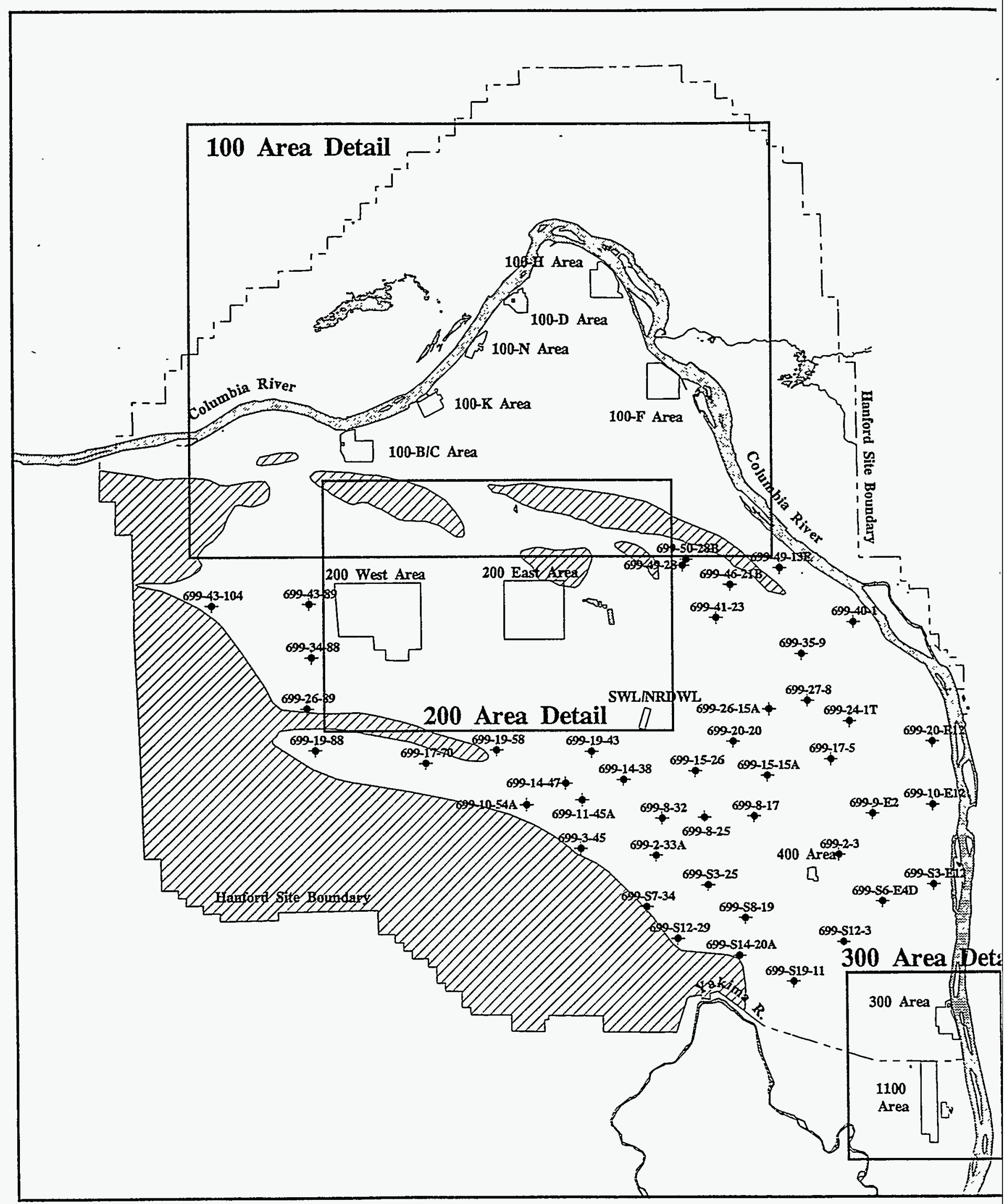




\section{Hanford Site} Index Map June 1994

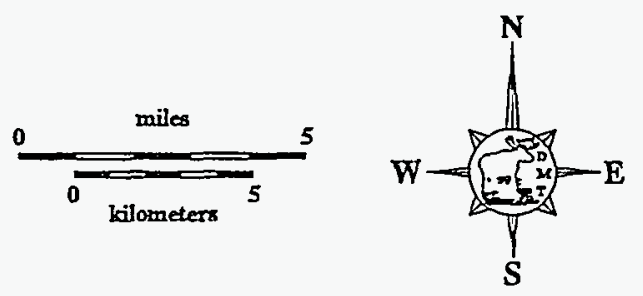

699-41-23

$-\quad$ Well name and location

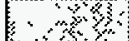

$\therefore$

Ponds, lakes, and rivers

Areas where basalt surface is generally above the water table.

Prepared by the Earth and Environmental Engineering Function, Westinghouse Hanford Company.

index_bs.mop-122094

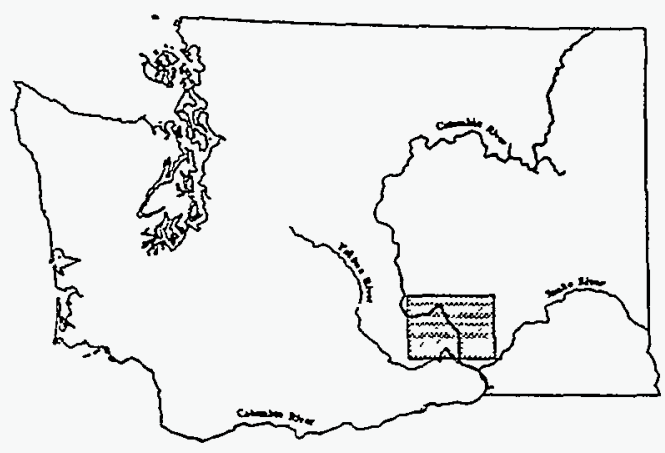

Figure-1. Hanford Site Location Map. 


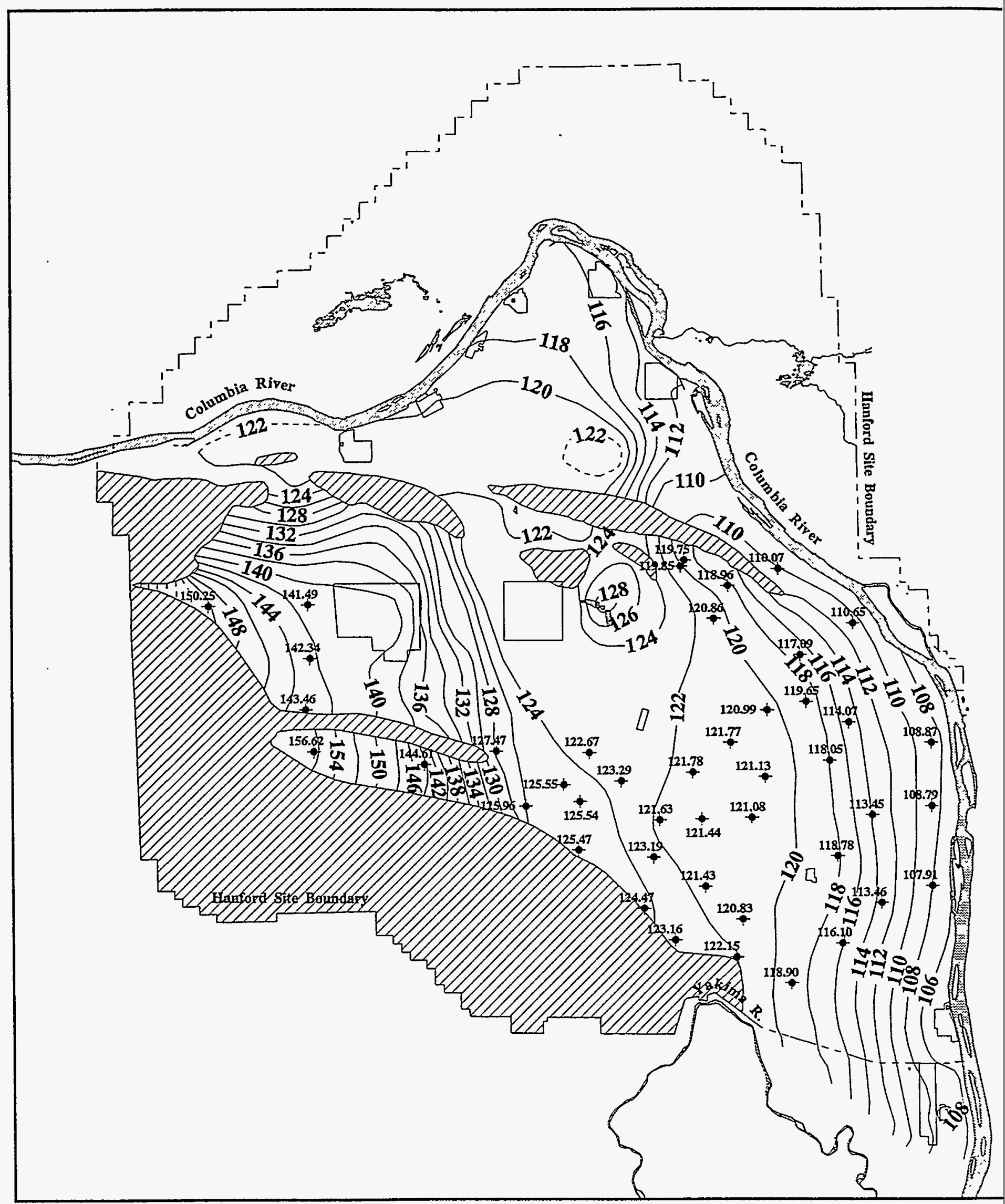




\section{Hanford Site Water Table June 1994

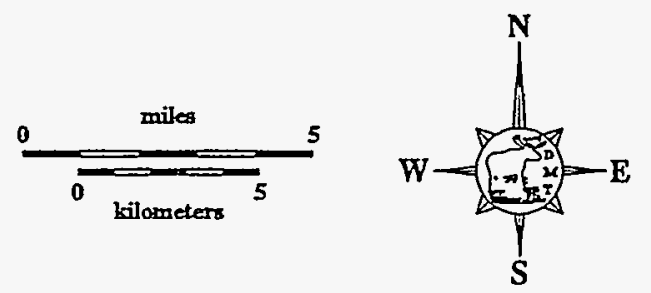

122.34

Water table elevation (m above msl)

122 - Water level contour (m above msl)

Ponds, lakes, and rivers

Areas where basalt surface is generally above the water table.

Prepared by the Earth and Environmental Engineering Function, Westinghouse Hanford Company.

wl hs.map-122194

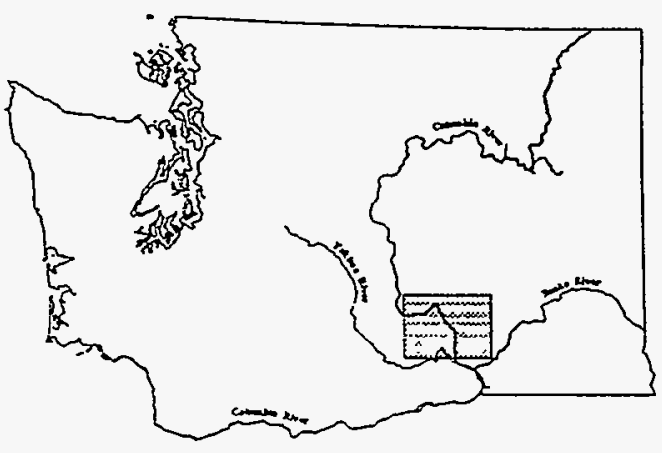

Figure 2. Hanford Site Water Table. 


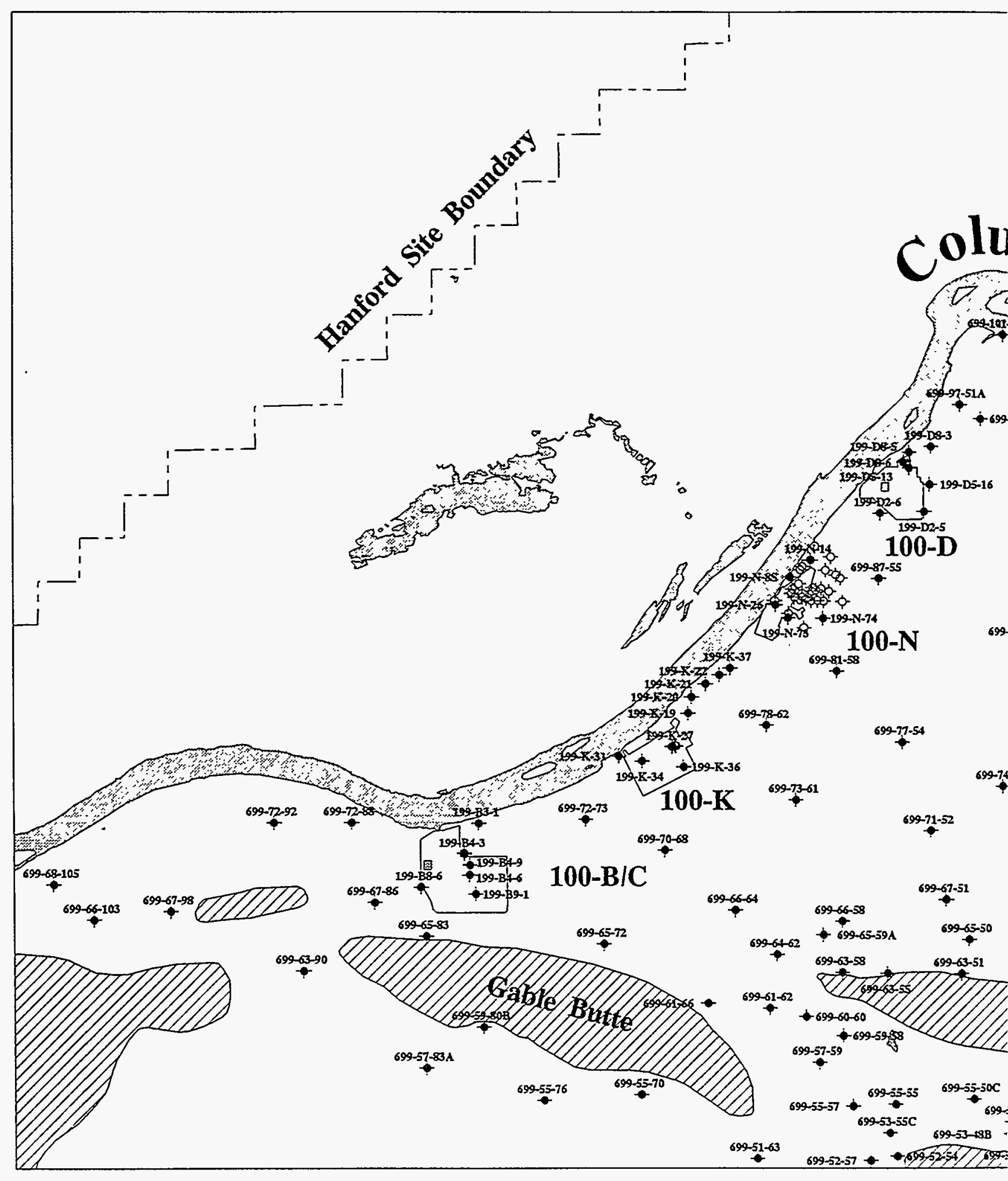




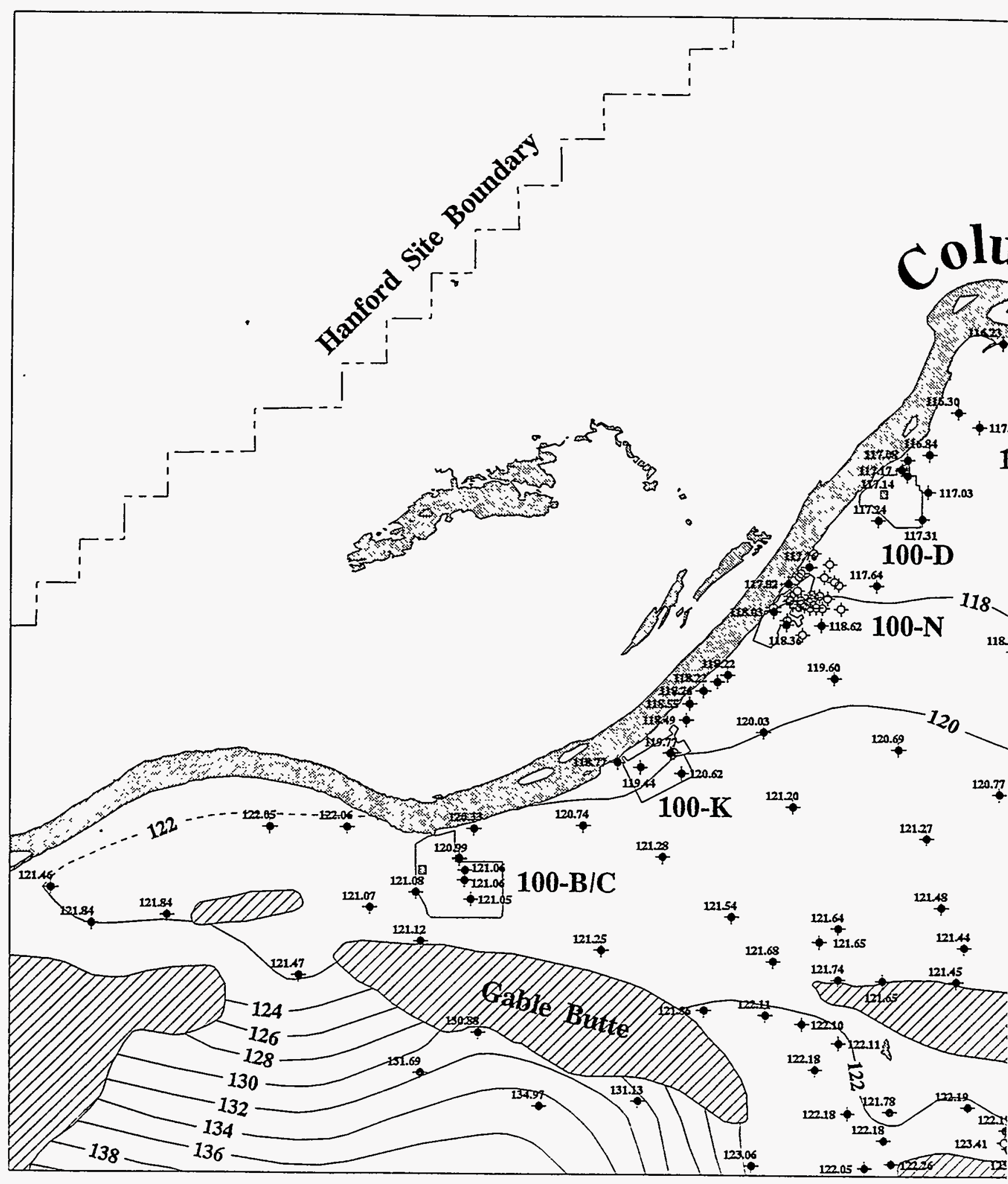




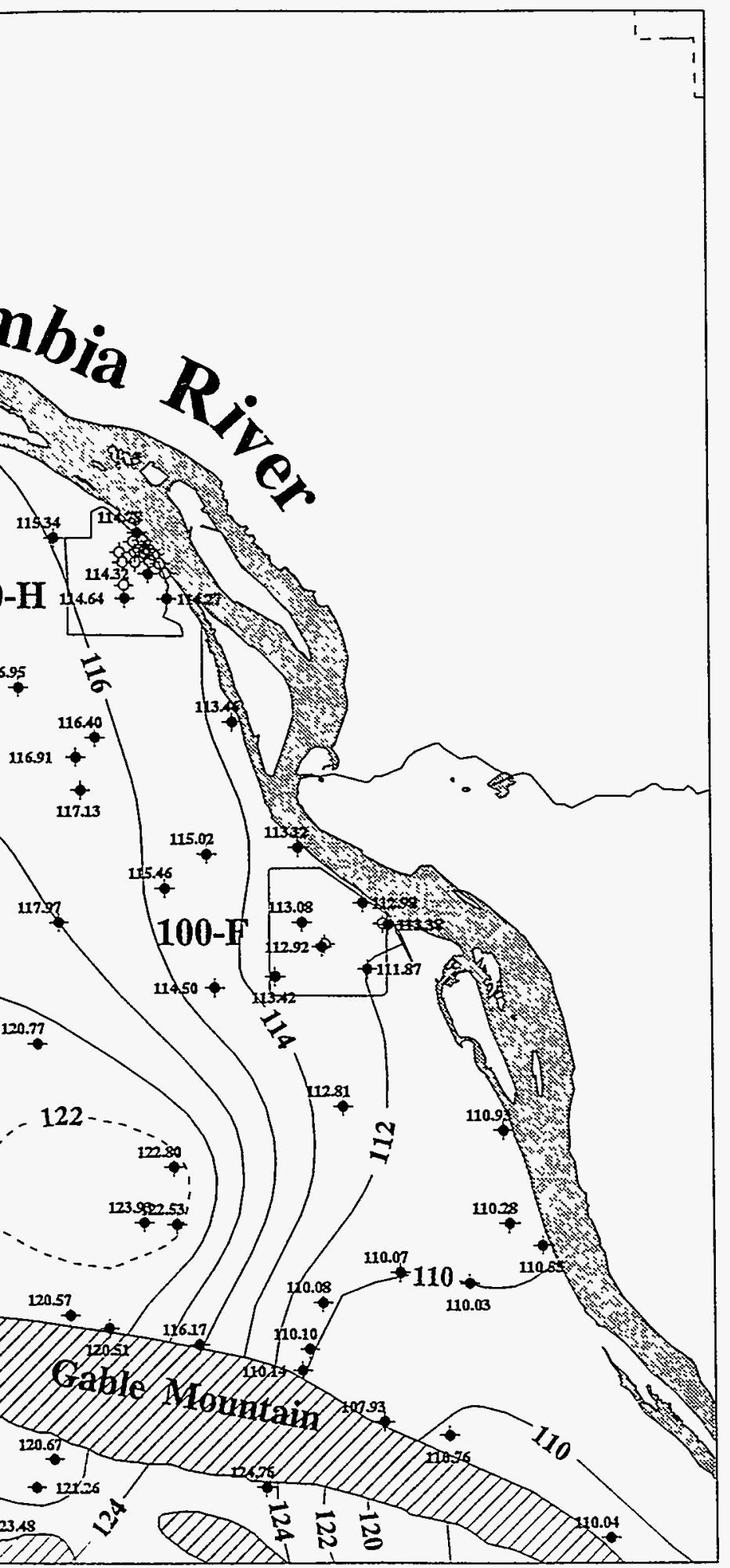

\section{Areas} Water Table June 1994

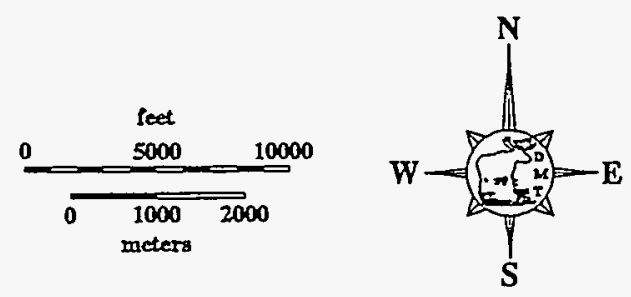

122.45

- Water table elevation (m above msl)

$\phi$ Well location

122- Water level contour (m above msl)

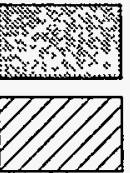

Ponds, lakes, and rivers

Areas where basalt surface is generally above the water table.

Prepared by the Earth and Environmental Engineering Function, Westinghouse Hanford Company.

FI_100_map-121794

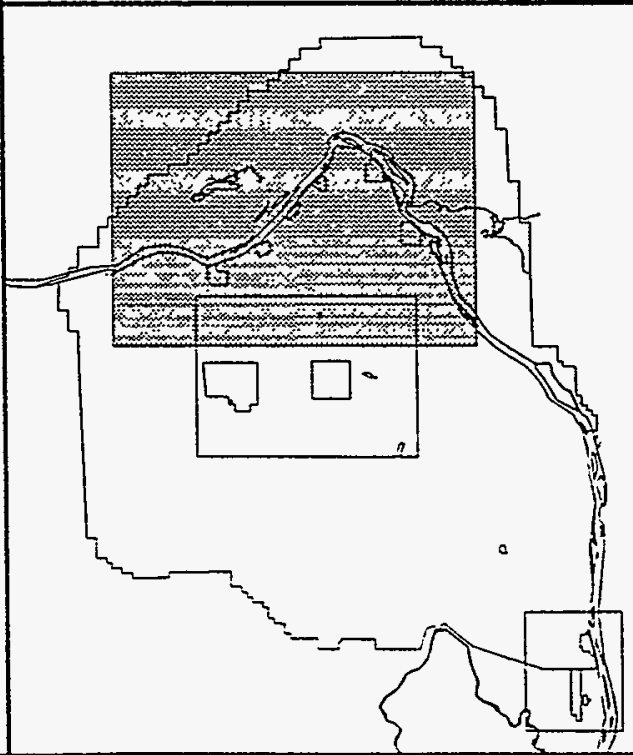

Figure 4. 100 Area Water Table. 


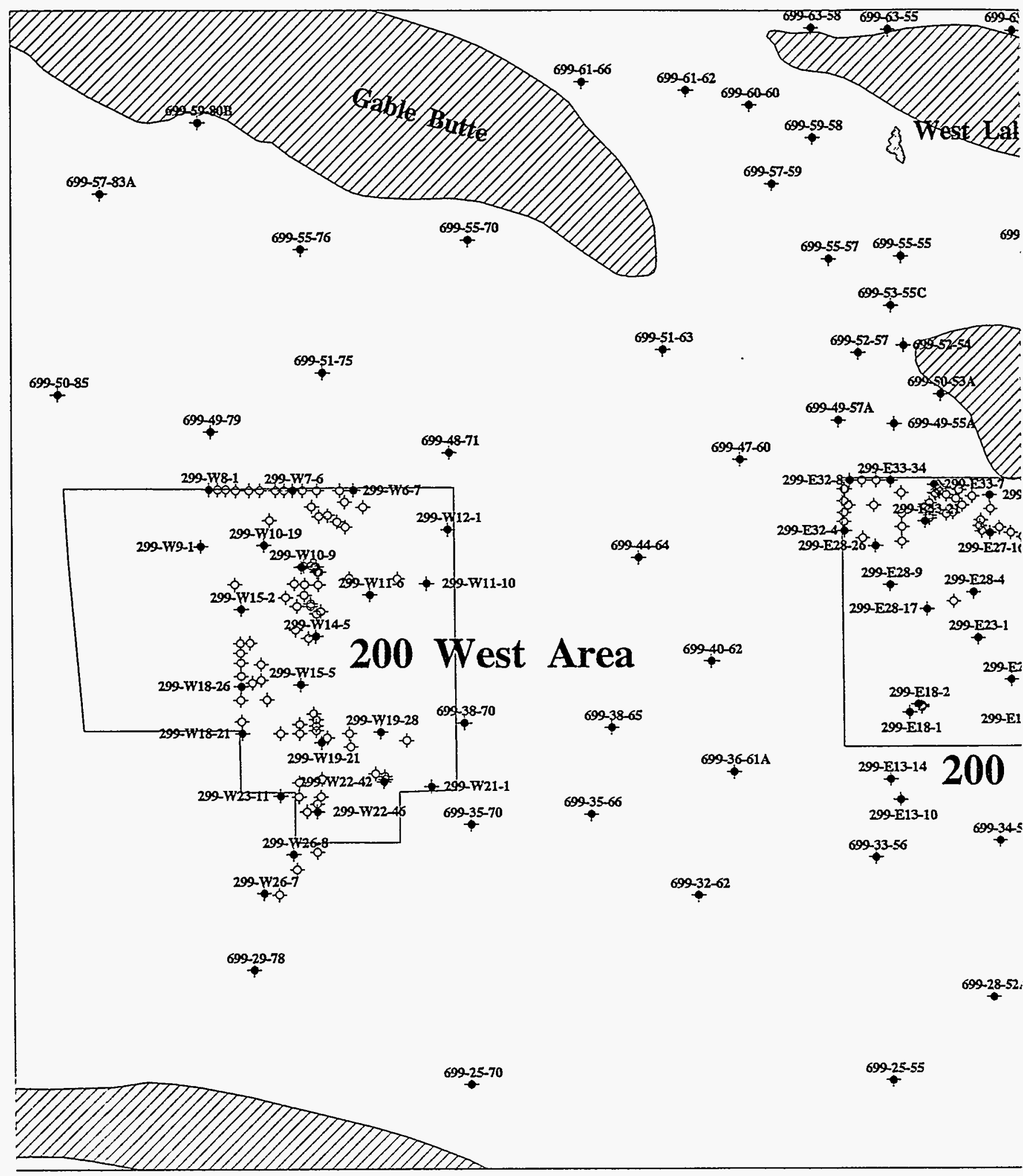




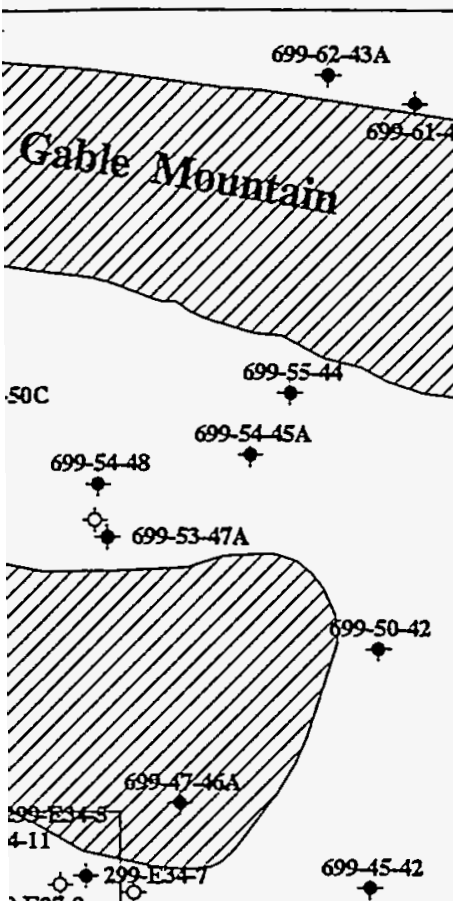

$2-0+298+3+1$

2E27-8 \& 299-E26-10

19927.

699-44-43B $+699-42 \mathrm{R}$. P.Ond

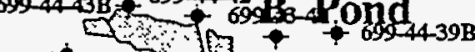

$$
\text { कو } 43-45
$$

E25-40 299-E 20-34 299-Eस

$+$$$
+
$$

$-209525-21$

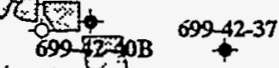

$+699430$
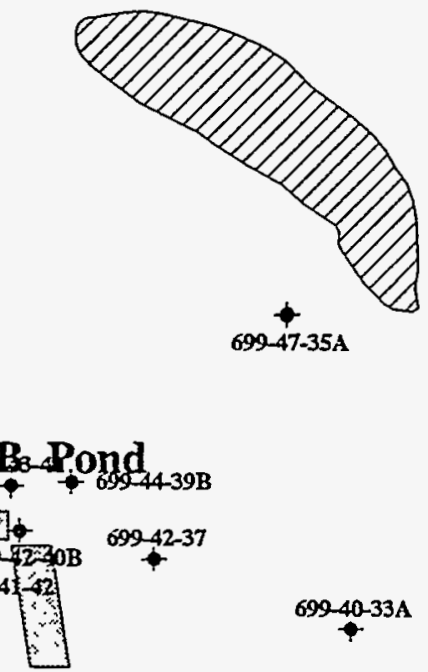

309-25-36

299-E17-18

ast Area

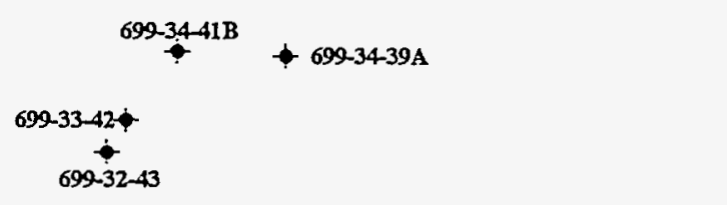

NRDWL/SWL

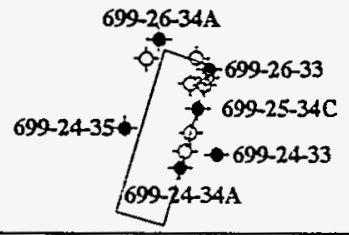

200 Areas

Index Map

June 1994
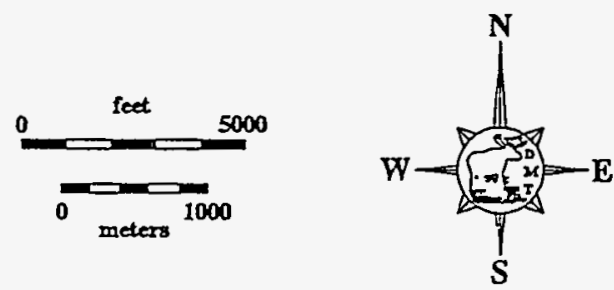

699-39-39

$+\quad$ Well name and location

- $-\quad$ Well location

Ponds, lakes, and rivers

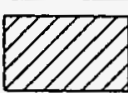

Areas where basalt surface is generally above the water table.

Prepared by the Earth and Environmental Engineering Function, Westinghouse Hanford Company.

index 200.map-122194

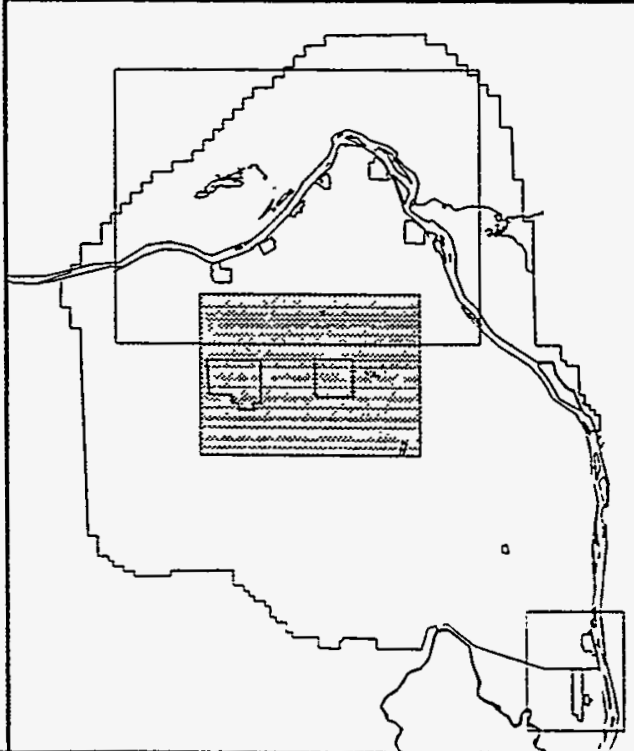

Figure 5. 200 Area Location Map. 


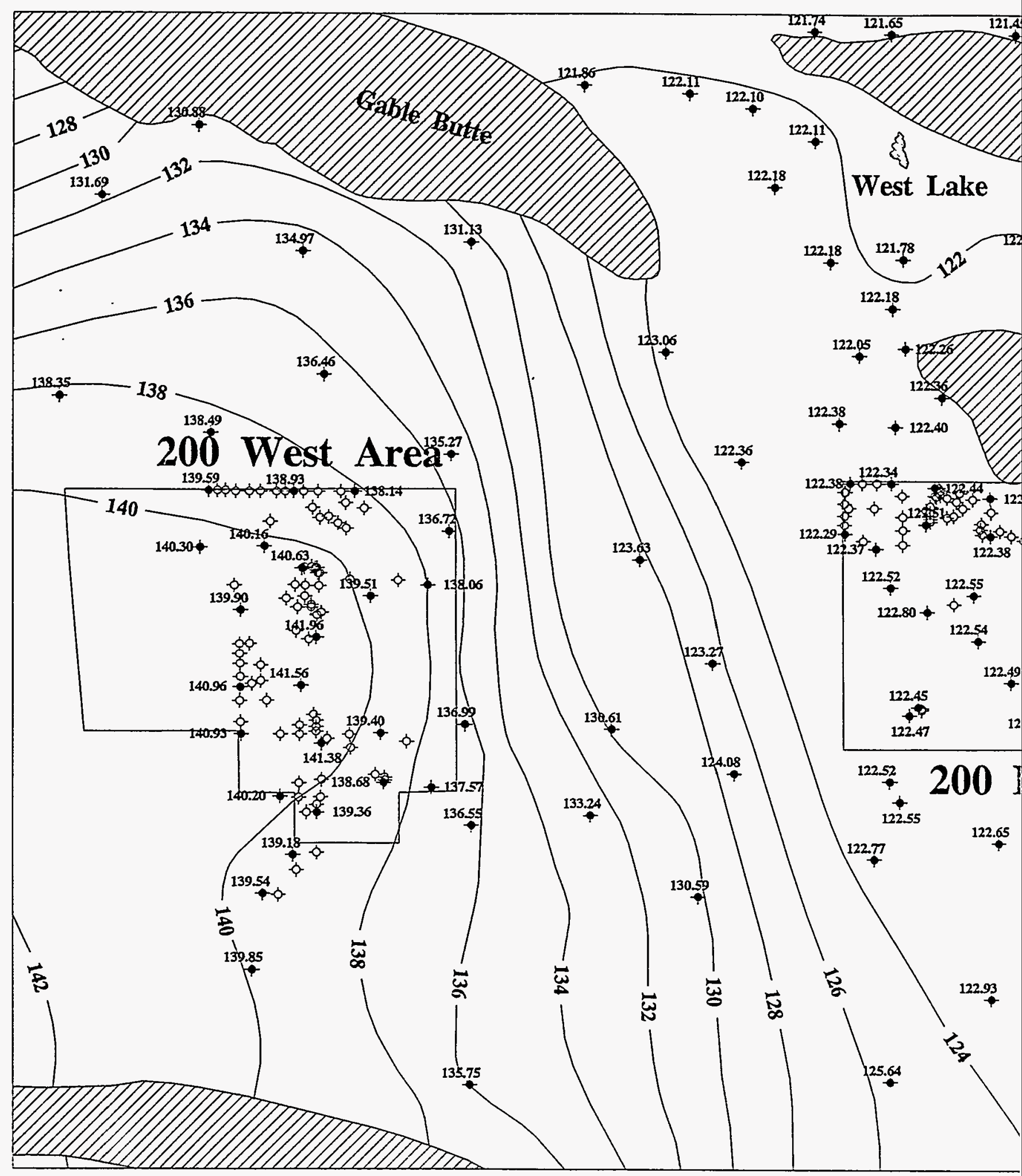




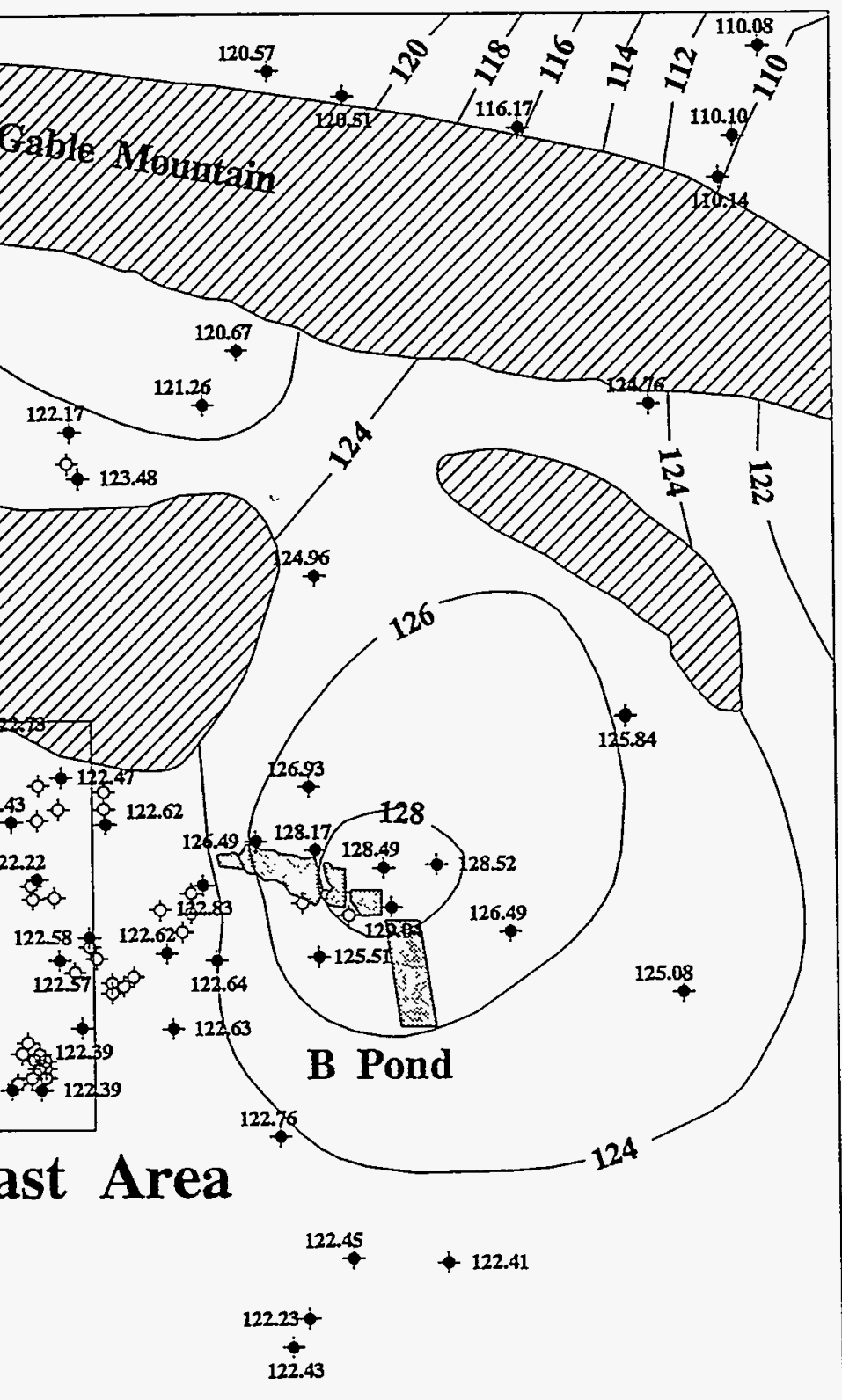

NRDWL/SWL

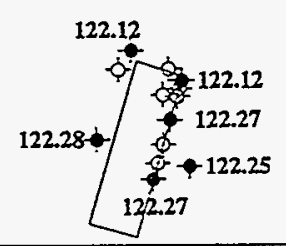

\section{Areas} Water Table June 1994

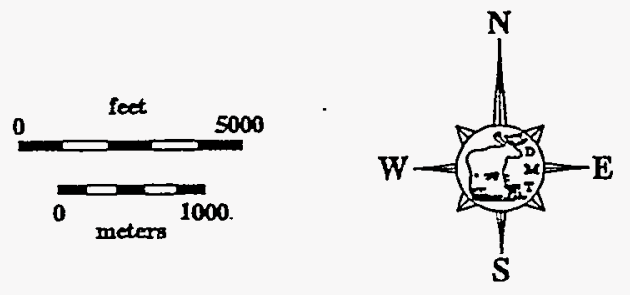

122.34

Water table elevation (m above msl)

-

-122 - Water level contour (m above $\mathrm{msl}$ )

3onds, lakes, and rivers

VIIT7 Areas where basalt surface is generally above the water table.

Prepared by the Earth and Environmental Engineering Function, Westinghouse Hanford Company.

v1 200.map-122194

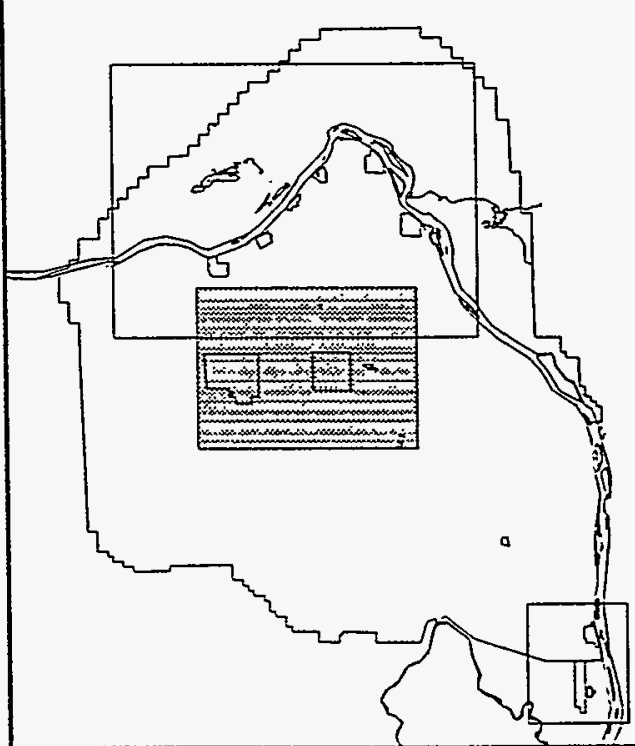

Figure 6. 200 Area Water Table. 


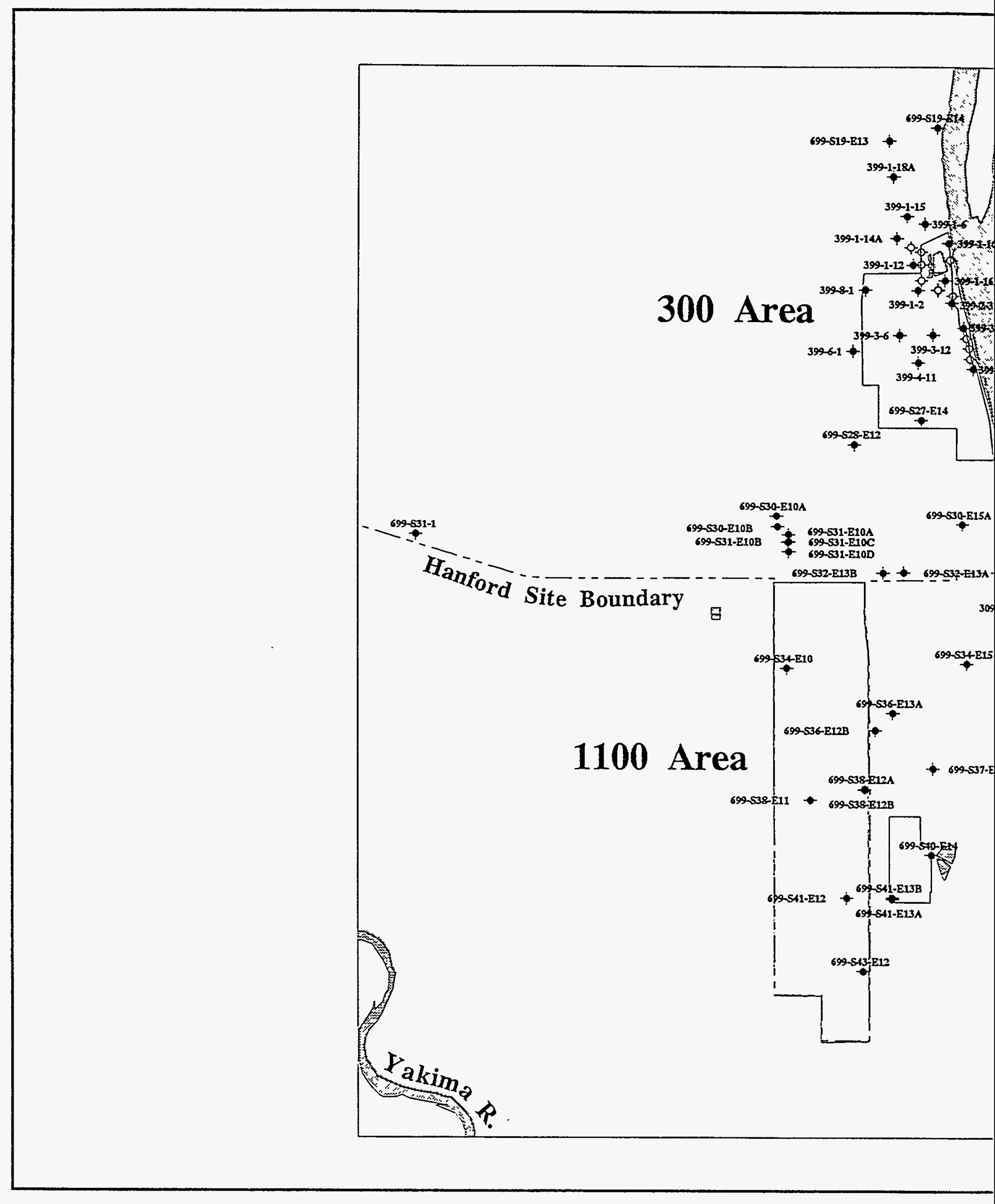


300 Area

Index Map

June 1994

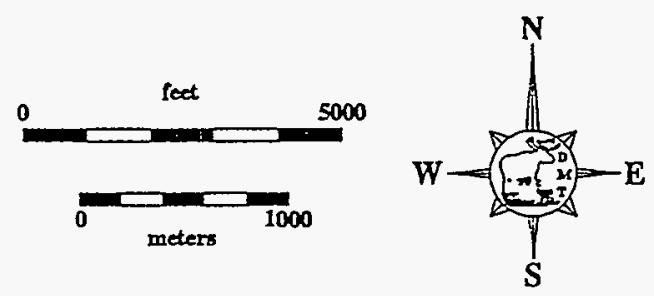

699-39-39

Well name and location

$-\phi-\quad$ Well location

Pि, Ponds, lakes, and rivers

P/PAPAPA

Areas where basalt surface is generally above the water table.

Prepared by the Earth and Environmental Engineering Function, Westinghouse Hanford Company.

index 300.map-122194

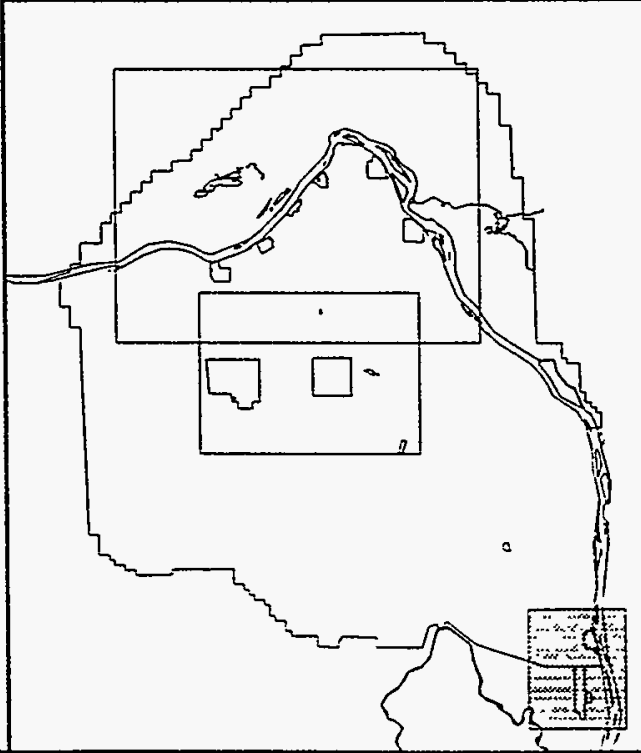

Figure 7. 300 Area Location Map. 


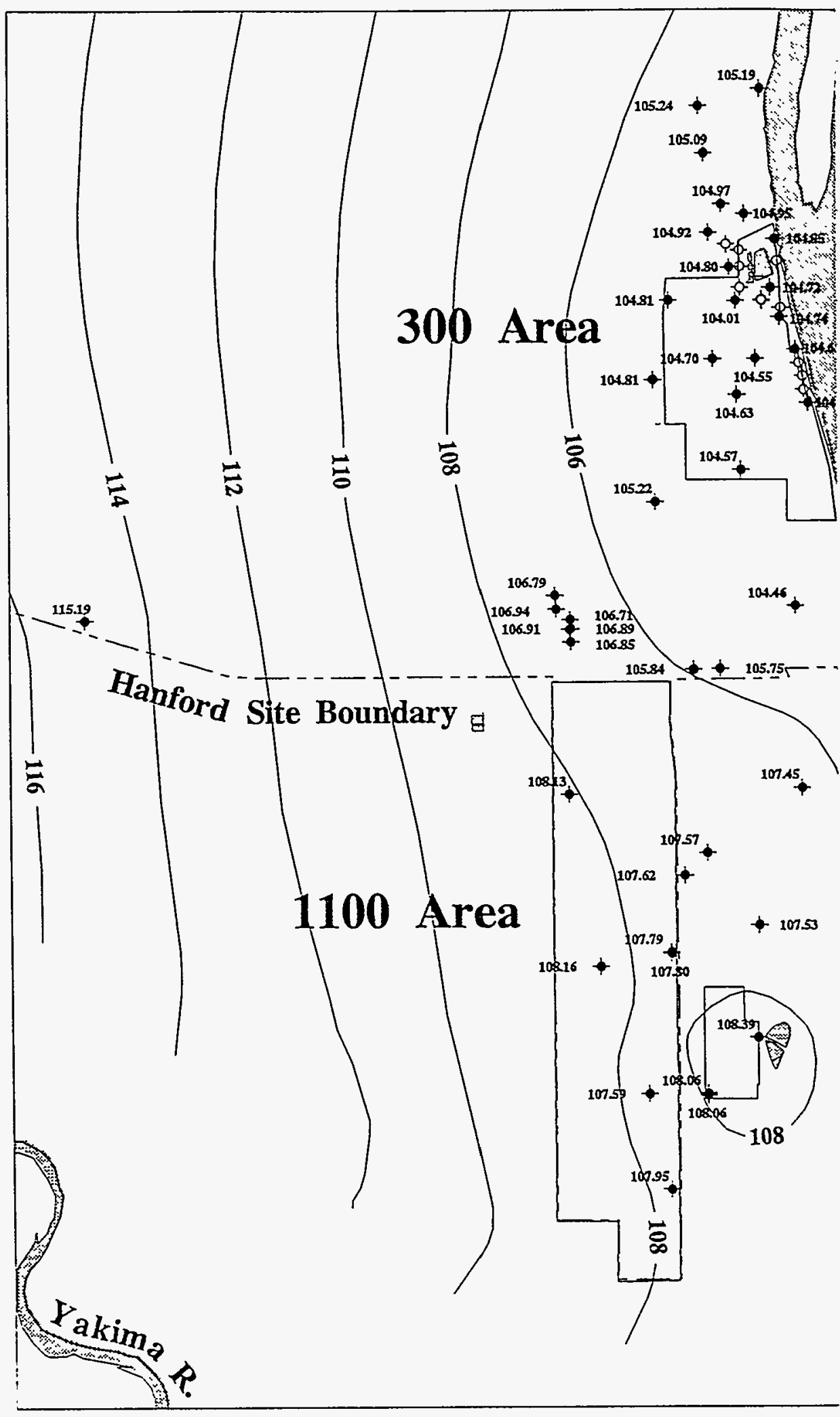




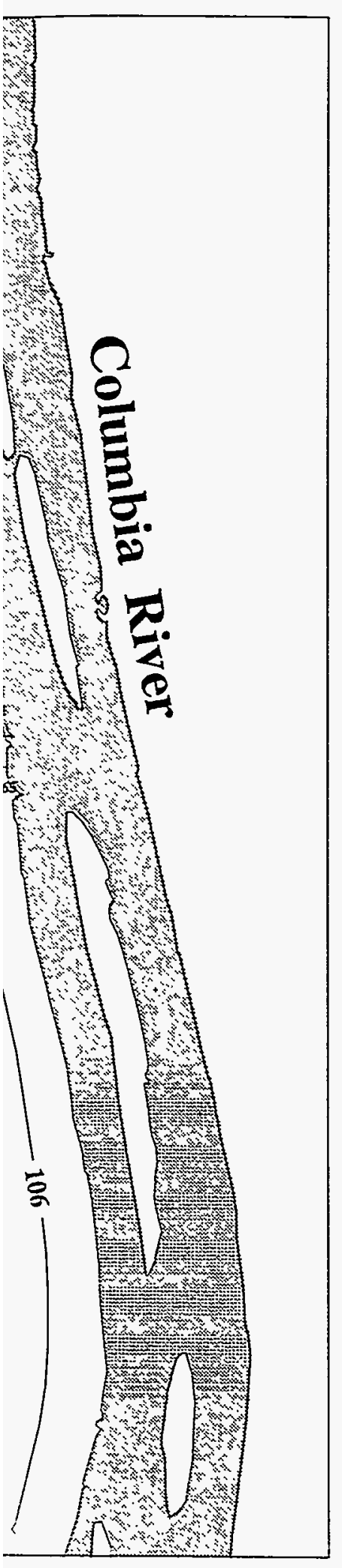

\section{Area}

Water Table

June 1994

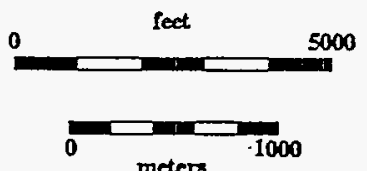

122.34

- Water table elevation

(m above msl)

-

$-122$

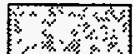

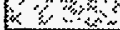

DPDA

Areas where basalt surface is generally above the water table.

Prepared by the Earth and Environmental Engineering Function, Westinghouse Hanford Company.

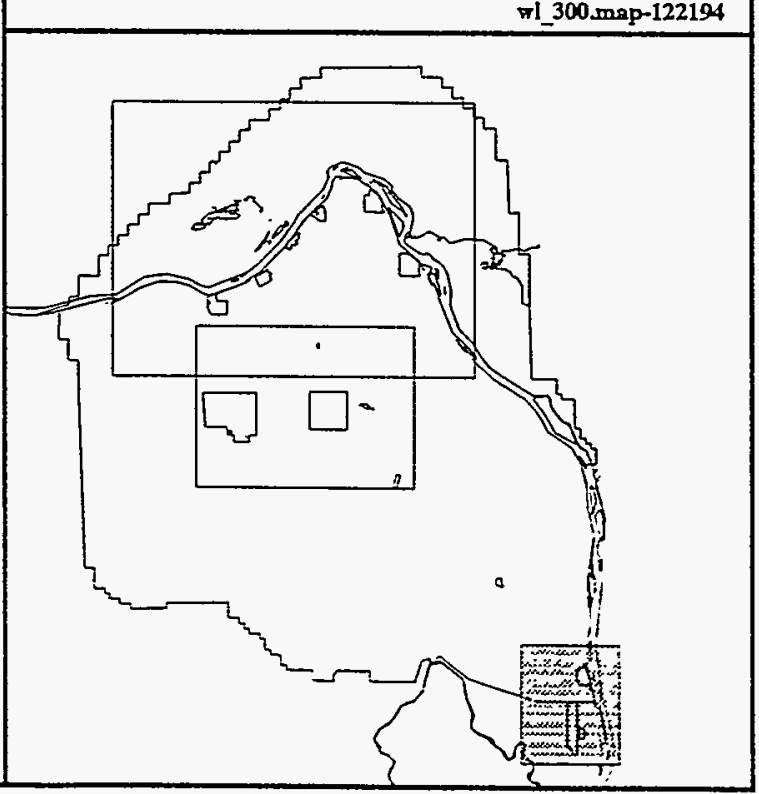

Figure 8. 300 Area Water Table. 
WHC-EP-0394-9

APPENDIX A

WATER LEVEL MEASUREMENT DATA 
WHC-EP-0394-9

This page intentionally left blank. 
Appendix A: June 1994 Water Level Measurement Data (Sheet 1 of 23)

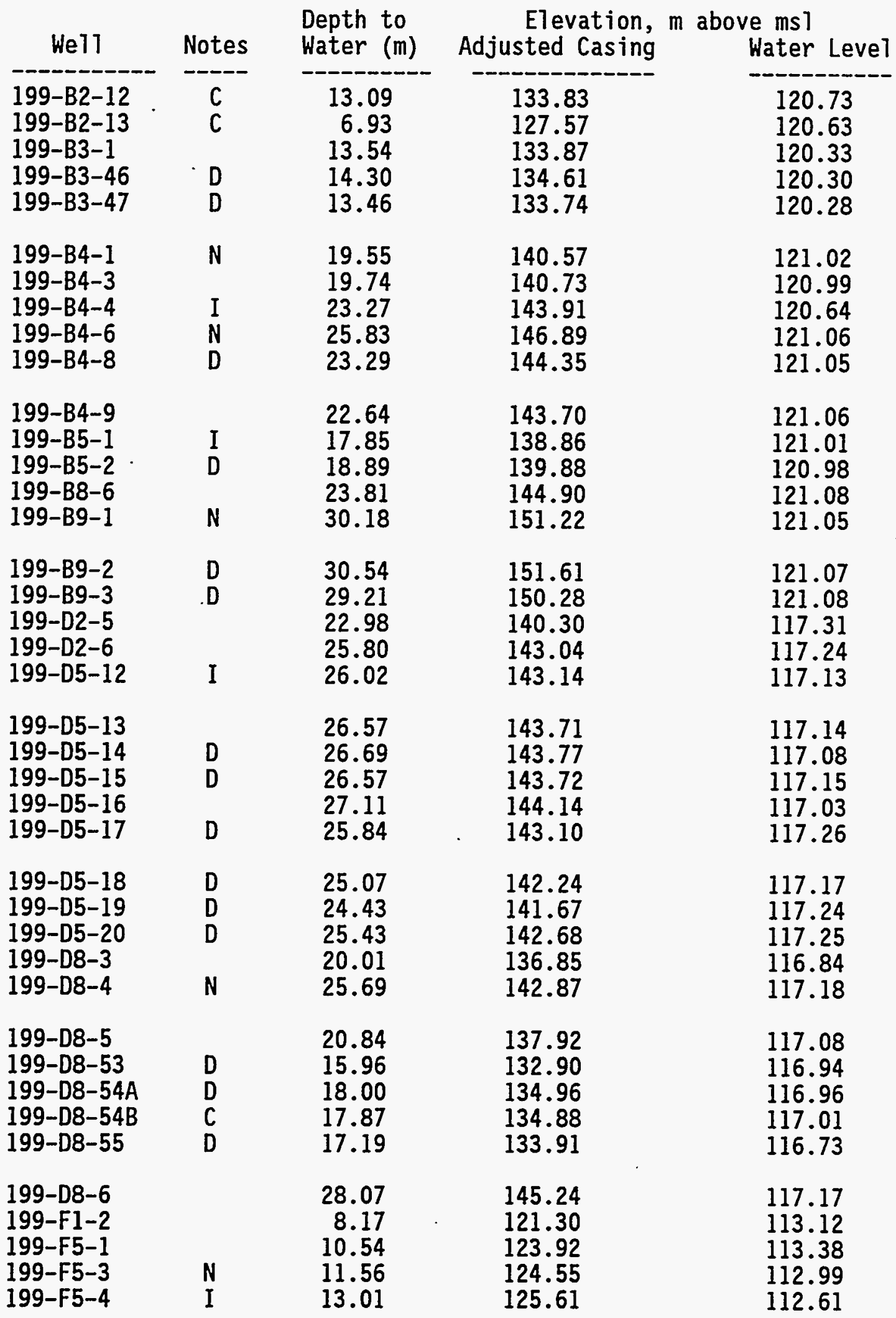


Appendix A: June 1994 Water Level Measurement Data (Sheet 2 of 23)

\begin{tabular}{|c|c|c|c|c|}
\hline Well & Notes & $\begin{array}{l}\text { Depth to } \\
\text { Water }(\mathrm{m})\end{array}$ & $\begin{array}{r}\text { Elevation, } \\
\text { Adjusted Casing }\end{array}$ & $\begin{array}{l}\text { m above ms } 1 \\
\text { Water Level }\end{array}$ \\
\hline $\begin{array}{l}199-F 5-42 \\
199-F 5-43 A \\
199-F 5-43 B \\
199-F 5-44 \\
199-F 5-45\end{array}$ & $\begin{array}{l}D \\
D \\
C \\
D \\
D\end{array}$ & $\begin{array}{r}6.07 \\
7.10 \\
7.16 \\
9.25 \\
13.57\end{array}$ & $\begin{array}{l}119.26 \\
120.50 \\
120.36 \\
122.73 \\
126.28\end{array}$ & $\begin{array}{l}113.19 \\
113.40 \\
113.19 \\
113.48 \\
112.72\end{array}$ \\
\hline $\begin{array}{l}199-F 5-46 \\
199-F 5-47 \\
199-F 5-48 \\
199-F 5-6 \\
199-F 6-1\end{array}$ & $\begin{array}{l}D \\
D \\
D \\
N \\
D\end{array}$ & $\begin{array}{l}14.25 \\
14.94 \\
14.47 \\
12.89 \\
10.82\end{array}$ & $\begin{array}{l}127.09 \\
127.72 \\
127.21 \\
125.87 \\
123.49\end{array}$ & $\begin{array}{l}112.83 \\
112.78 \\
112.74 \\
112.98 \\
112.67\end{array}$ \\
\hline $\begin{array}{l}199-F 7-1 \\
199-F 7-2 \\
199-F 7-3 \\
199-F 8-1 \\
199-F 8-2\end{array}$ & $\begin{array}{l}N \\
D\end{array}$ & $\begin{array}{r}5.38 \\
7.17 \\
6.78 \\
10.79 \\
12.32\end{array}$ & $\begin{array}{l}118.79 \\
120.25 \\
120.35 \\
123.71 \\
125.19\end{array}$ & $\begin{array}{l}113.42 \\
113.08 \\
113.56 \\
112.92 \\
112.88\end{array}$ \\
\hline $\begin{array}{l}199-F 8-3 \\
199-F 8-4 \\
199-H 3-1 \\
199-H 3-2 A \\
199-H 3-2 B\end{array}$ & $\begin{array}{l}\mathrm{N} \\
\mathrm{N} \\
\mathrm{N}\end{array}$ & $\begin{array}{r}8.70 \\
13.38 \\
13.97 \\
12.86 \\
13.05\end{array}$ & $\begin{array}{l}121.83 \\
125.25 \\
128.47 \\
127.35 \\
127.53\end{array}$ & $\begin{array}{l}113.14 \\
111.87 \\
114.50 \\
114.50 \\
114.49\end{array}$ \\
\hline $\begin{array}{l}199-\mathrm{H} 3-2 \mathrm{C} \\
199-\mathrm{H} 4-10 \\
199-\mathrm{H} 4-11 \\
199-\mathrm{H} 4-12 \mathrm{~A} \\
199-\mathrm{H} 4-12 \mathrm{~B}\end{array}$ & $\begin{array}{l}\mathrm{C} \\
N \\
N \\
N \\
N\end{array}$ & $\begin{array}{r}12.96 \\
8.49 \\
12.35 \\
11.29 \\
11.30\end{array}$ & $\begin{array}{l}127.47 \\
123.27 \\
127.05 \\
126.03 \\
126.04\end{array}$ & $\begin{array}{l}114.52 \\
114.78 \\
114.70 \\
114.75 \\
114.74\end{array}$ \\
\hline $\begin{array}{l}199-H 4-12 C \\
199-H 4-13 \\
199-H 4-14 \\
199-H 4-15 A \\
199-H 4-15 B\end{array}$ & $\begin{array}{l}\mathrm{C} \\
\mathrm{N} \\
\mathrm{N} \\
\mathrm{N} \\
\mathrm{N}\end{array}$ & $\begin{array}{r}11.34 \\
12.83 \\
13.73 \\
9.35 \\
9.27\end{array}$ & $\begin{array}{l}126.04 \\
127.47 \\
128.20 \\
124.12 \\
124.03\end{array}$ & $\begin{array}{l}114.70 \\
114.64 \\
114.46 \\
114.77 \\
114.76\end{array}$ \\
\hline $\begin{array}{l}199-H 4-15 C R \\
199-H 4-15 C S \\
199-H 4-16 \\
199-H 4-17 \\
199-H 4-18\end{array}$ & $\begin{array}{l}C \\
C \\
N \\
N\end{array}$ & $\begin{array}{r}9.96 \\
9.43 \\
14.99 \\
13.16 \\
14.12\end{array}$ & $\begin{array}{l}124.17 \\
124.19 \\
129.31 \\
127.74 \\
128.57\end{array}$ & $\begin{array}{l}114.21 \\
114.76 \\
114.32 \\
114.57 \\
114.45\end{array}$ \\
\hline
\end{tabular}


Appendix A: June 1994 Water Level Measurement Data

(Sheet 3 of 23)

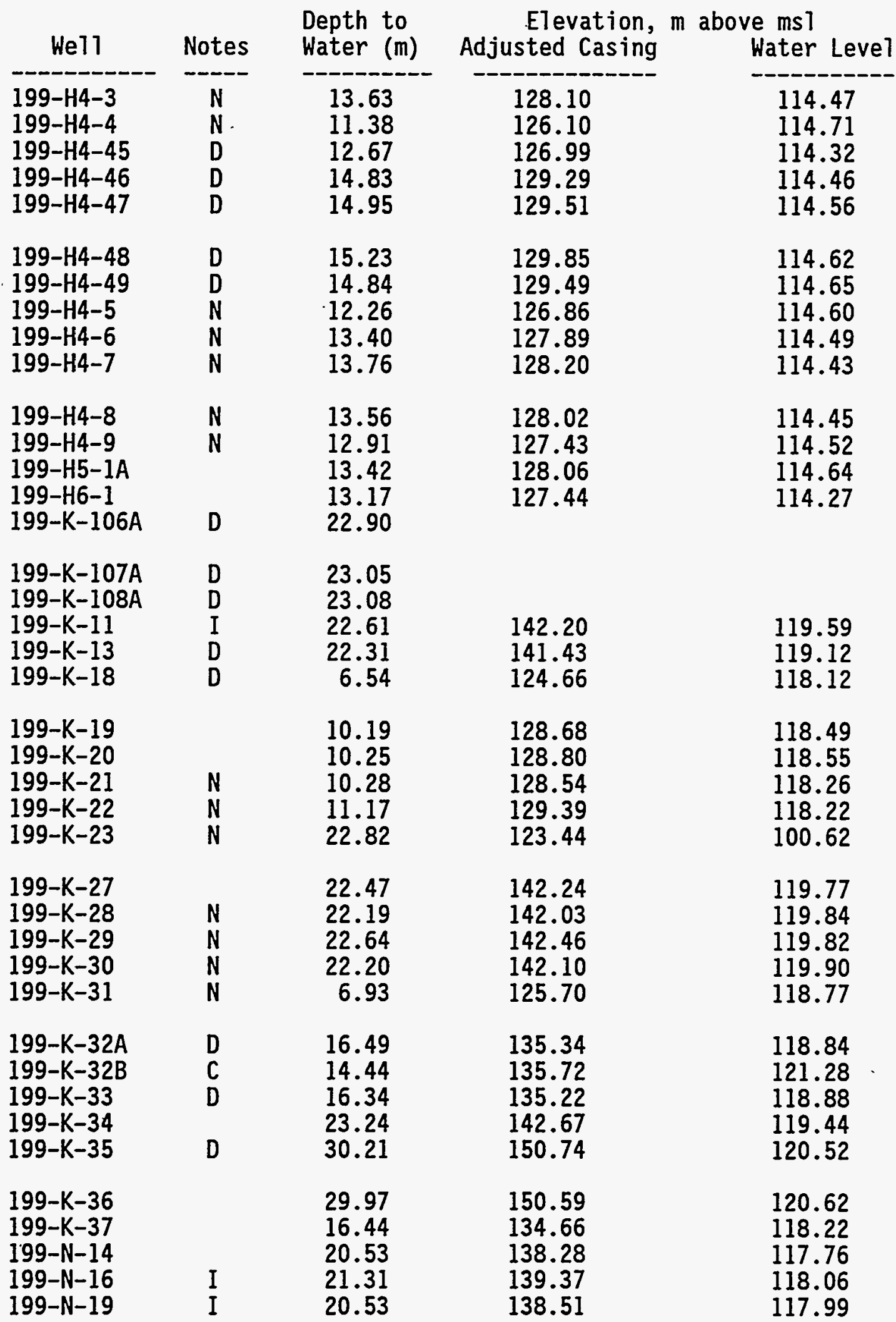


Appendix A: June 1994 Water Level Measurement Data (Sheet 4 of 23)

\begin{tabular}{|c|c|c|c|c|}
\hline Hell & Notes & $\begin{array}{l}\text { Depth to } \\
\text { Water (m) }\end{array}$ & $\begin{array}{r}\text { Elevation, } \\
\text { Adjusted Casing }\end{array}$ & $\begin{array}{l}\mathrm{m} \text { above } \mathrm{ms}\rceil \\
\text { Water Leve } 1\end{array}$ \\
\hline $\begin{array}{l}199-N-2 \\
199-N-20 \\
199-N-21 \\
199-N-23 \\
199-N-25\end{array}$ & $\begin{array}{l}\mathrm{I} \\
\mathrm{I} \\
\mathrm{I} \\
\mathrm{I} \\
\mathrm{N}\end{array}$ & $\begin{array}{l}22.37 \\
21.09 \\
21.43 \\
21.20 \\
11.89\end{array}$ & $\begin{array}{l}140.13 \\
139.10 \\
139.44 \\
139.23 \\
129.92\end{array}$ & $\begin{array}{l}117.77 \\
118.00 \\
118.01 \\
118.03 \\
118.03\end{array}$ \\
\hline $\begin{array}{l}199-N-26 \\
199-N-27 \\
199-N-28 \\
199-N-29 \\
199-N-3\end{array}$ & $\begin{array}{l}N \\
N \\
N \\
I\end{array}$ & $\begin{array}{l}21.04 \\
18.91 \\
23.35 \\
23.66 \\
22.19\end{array}$ & $\begin{array}{l}139.07 \\
137.04 \\
141.65 \\
141.96 \\
140.02\end{array}$ & $\begin{array}{l}118.03 \\
118.13 \\
118.31 \\
118.30 \\
117.84\end{array}$ \\
\hline $\begin{array}{l}199-N-31 \\
199-N-32 \\
199-N-33 \\
199-N-34 \\
199-N-41\end{array}$ & $\begin{array}{l}N \\
N \\
N \\
N \\
N\end{array}$ & $\begin{array}{l}23.19 \\
22.96 \\
22.38 \\
22.19 \\
21.97\end{array}$ & $\begin{array}{l}141.01 \\
141.00 \\
140.32 \\
140.25 \\
139.63\end{array}$ & $\begin{array}{l}117.82 \\
118.03 \\
117.94 \\
118.07 \\
117.66\end{array}$ \\
\hline $\begin{array}{l}199-N-42 \\
199-N-49 \\
199-N-50 \\
199-N-51 \\
199-N-52\end{array}$ & $\begin{array}{l}N \\
N \\
N \\
N \\
N\end{array}$ & $\begin{array}{l}21.13 \\
20.06 \\
23.80 \\
23.29 \\
23.21\end{array}$ & $\begin{array}{l}138.88 \\
137.54 \\
141.40 \\
141.04 \\
141.49\end{array}$ & $\begin{array}{l}117.74 \\
117.49 \\
117.60 \\
117.75 \\
118.28\end{array}$ \\
\hline $\begin{array}{l}199-N-54 \\
199-N-55 \\
199-N-56 \\
199-N-57 \\
199-N-62\end{array}$ & $\begin{array}{l}N \\
N \\
N \\
N \\
N\end{array}$ & $\begin{array}{l}21.70 \\
21.80 \\
21.95 \\
21.59 \\
23.15\end{array}$ & $\begin{array}{l}139.60 \\
139.71 \\
139.78 \\
139.68 \\
141.46\end{array}$ & $\begin{array}{l}117.90 \\
117.91 \\
117.82 \\
118.09 \\
118.31\end{array}$ \\
\hline $\begin{array}{l}199-N-63 \\
199-N-64 \\
199-N-65 \\
199-N-67 \\
199-N-69\end{array}$ & $\begin{array}{l}N \\
N \\
N \\
N \\
C\end{array}$ & $\begin{array}{l}24.17 \\
20.60 \\
21.27 \\
22.12 \\
22.23\end{array}$ & $\begin{array}{l}142.41 \\
138.73 \\
139.29 \\
139.89 \\
140.01\end{array}$ & $\begin{array}{l}118.24 \\
118.13 \\
118.02 \\
117.78 \\
117.78\end{array}$ \\
\hline $\begin{array}{l}199-N-70 \\
199-N-71 \\
199-N-72 \\
199-N-73 \\
199-N-74\end{array}$ & $\begin{array}{l}C \\
N \\
N\end{array}$ & $\begin{array}{l}20.84 \\
22.43 \\
21.33 \\
22.84 \\
20.87\end{array}$ & $\begin{array}{l}138.59 \\
141.13 \\
139.90 \\
141.20 \\
139.49\end{array}$ & $\begin{array}{l}117.75 \\
118.70 \\
118.57 \\
118.36 \\
118.62\end{array}$ \\
\hline $\begin{array}{l}199-N-75 \\
199-N-76 \\
199-N-77 \\
199-N-80 \\
199-N-81\end{array}$ & $\begin{array}{l}N \\
N \\
N \\
C \\
D\end{array}$ & $\begin{array}{l}21.42 \\
20.01 \\
21.55 \\
21.55 \\
23.25\end{array}$ & $\begin{array}{l}139.23 \\
137.81 \\
140.04 \\
139.50 \\
141.03\end{array}$ & $\begin{array}{l}117.80 \\
117.80 \\
118.49 \\
117.96 \\
117.78\end{array}$ \\
\hline
\end{tabular}


Appendix A: June 1994 Water Level Measurement Data (Sheet 5 of 23)

\begin{tabular}{|c|c|c|c|c|}
\hline We 11 & Notes & $\begin{array}{l}\text { Depth to } \\
\text { Water (m) }\end{array}$ & $\begin{array}{r}\text { Elevation, } \\
\text { Adjusted Casing }\end{array}$ & $\begin{array}{l}\text { m above msl } \\
\text { Water Level }\end{array}$ \\
\hline $\begin{array}{l}199-N-8 S \\
299-E 13-10 \\
299-E 13-12 \\
299-E 13-14 \\
299-E 16-1\end{array}$ & $c$ & $\begin{array}{r}5.66 \\
102.65 \\
100.94 \\
104.60 \\
88.26\end{array}$ & $\begin{array}{l}123.48 \\
225.20 \\
222.91 \\
227.12 \\
212.27\end{array}$ & $\begin{array}{l}117.82 \\
122.55 \\
121.97 \\
122.52 \\
124.02\end{array}$ \\
\hline $\begin{array}{l}299-E 17-1 \\
299-E 17-10 \\
299-E 17-12 \\
299-E 17-13 \\
299-E 17-14\end{array}$ & & $\begin{array}{l}96.90 \\
95.47 \\
97.53 \\
96.88 \\
97.73\end{array}$ & $\begin{array}{l}219.20 \\
217.85 \\
219.97 \\
219.23 \\
220.12\end{array}$ & $\begin{array}{l}122.30 \\
122.38 \\
122.44 \\
122.35 \\
122.39\end{array}$ \\
\hline $\begin{array}{l}299-E 17-15 \\
299-E 17-16 \\
299-E 17-17 \\
299-E 17-18 \\
299-E 17-5\end{array}$ & & $\begin{array}{l}97.88 \\
97.34 \\
97.10 \\
97.27 \\
96.82\end{array}$ & $\begin{array}{l}220.00 \\
219.63 \\
219.43 \\
219.65 \\
219.06\end{array}$ & $\begin{array}{l}122.12 \\
122.29 \\
122.33 \\
122.39 \\
122.24\end{array}$ \\
\hline $\begin{array}{l}299-E 17-6 \\
299-E 17-8 \\
299-E 17-9 \\
299-E 18-1 \\
299-E 18-2\end{array}$ & I & $\begin{array}{l}97.11 \\
96.70 \\
96.41 \\
97.06 \\
97.44\end{array}$ & $\begin{array}{l}219.49 \\
218.96 \\
218.74 \\
219.53 \\
219.89\end{array}$ & $\begin{array}{l}122.37 \\
122.26 \\
122.33 \\
122.47 \\
122.45\end{array}$ \\
\hline $\begin{array}{l}299-E 18-3 \\
299-E 18-4 \\
299-E 23-1 \\
299-E 23-2 \\
299-E 24-16\end{array}$ & I & $\begin{array}{l}97.76 \\
97.47 \\
94.82 \\
97.18 \\
96.60\end{array}$ & $\begin{array}{l}220.09 \\
219.93 \\
217.35 \\
219.65 \\
218.93\end{array}$ & $\begin{array}{l}122.33 \\
122.47 \\
122.54 \\
122.47 \\
122.33\end{array}$ \\
\hline $\begin{array}{l}299-E 24-18 \\
299-E 24-2 \\
299-E 24-20 \\
299-E 24-7 \\
299-E 24-8\end{array}$ & I & $\begin{array}{l}96.81 \\
96.28 \\
87.52 \\
95.75 \\
87.37\end{array}$ & $\begin{array}{l}219.24 \\
218.68 \\
210.09 \\
218.24 \\
209.95\end{array}$ & $\begin{array}{l}122.42 \\
122.40 \\
122.57 \\
122.49 \\
122.58\end{array}$ \\
\hline $\begin{array}{l}299-E 25-10 \\
299-E 25-11 \\
299-E 25-18 \\
299-E 25-19 \\
299-E 25-2\end{array}$ & $\begin{array}{l}I \\
I \\
C \\
C\end{array}$ & $\begin{array}{l}77.38 \\
85.16 \\
84.45 \\
83.94 \\
83.33\end{array}$ & $\begin{array}{l}199.90 \\
207.65 \\
206.97 \\
206.41 \\
205.94\end{array}$ & $\begin{array}{l}122.52 \\
122.49 \\
122.52 \\
122.47 \\
122.61\end{array}$ \\
\hline
\end{tabular}


Appendix A: June 1994 Water Level Measurement Data (Sheet 6 of 23)

\begin{tabular}{|c|c|c|c|c|}
\hline Welt & Notes & $\begin{array}{l}\text { Depth to } \\
\text { Water (m) }\end{array}$ & $\begin{array}{l}\text { Elevation, } \\
\text { Adjusted Casing }\end{array}$ & $\begin{array}{l}\text { m above ms } 7 \\
\text { Water Level }\end{array}$ \\
\hline $\begin{array}{l}299-E 25-20 \\
299-E 25-21 \\
299-E 25-26 \\
299-E 25-28 \\
299-E 25-32 P\end{array}$ & $\begin{array}{l}\text { C } \\
I\end{array}$ & $\begin{array}{l}83.66 \\
83.80 \\
81.18 \\
79.26 \\
81.59\end{array}$ & $\begin{array}{l}206.14 \\
206.43 \\
203.76 \\
201.91 \\
204.23\end{array}$ & $\begin{array}{l}122.47 \\
122.63 \\
122.58 \\
122.65 \\
122.64\end{array}$ \\
\hline $\begin{array}{l}\text { 299-E25-320 } \\
299-E 25-34 \\
299-E 25-35 \\
299-E 25-36 \\
299-E 25-40\end{array}$ & I & $\begin{array}{l}81.50 \\
79.42 \\
83.06 \\
93.22 \\
80.39\end{array}$ & $\begin{array}{l}204.23 \\
202.04 \\
205.55 \\
215.61 \\
202.97\end{array}$ & $\begin{array}{l}122.72 \\
122.62 \\
122.50 \\
122.39 \\
122.58\end{array}$ \\
\hline $\begin{array}{l}299-E 25-41 \\
299-E 25-42 \\
299-E 25-43 \\
299-E 25-46 \\
299-E 25-47\end{array}$ & & $\begin{array}{l}82.06 \\
85.74 \\
75.40 \\
89.22 \\
82.77\end{array}$ & $\begin{array}{l}204.66 \\
208.27 \\
198.09 \\
211.78 \\
205.37\end{array}$ & $\begin{array}{l}122.61 \\
122.53 \\
122.69 \\
122.55 \\
122.60\end{array}$ \\
\hline $\begin{array}{l}299-E 25-48 \\
299-E 25-9 \\
299-E 26-1 \\
299-E 26-10 \\
299-E 26-12\end{array}$ & I & $\begin{array}{l}85.39 \\
77.70 \\
65.49 \\
60.71 \\
69.52\end{array}$ & $\begin{array}{l}207.97 \\
199.60 \\
188.14 \\
183.33 \\
192.25\end{array}$ & $\begin{array}{l}122.58 \\
121.90 \\
122.65 \\
122.62 \\
122.73\end{array}$ \\
\hline $\begin{array}{l}299-E 26-13 \\
299-E 26-2 \\
299-E 26-4 \\
299-E 26-8 \\
299-E 26-9\end{array}$ & $\begin{array}{l}\text { I } \\
\text { C }\end{array}$ & $\begin{array}{l}61.70 \\
71.02 \\
74.84 \\
66.27 \\
61.15\end{array}$ & $\begin{array}{l}184.41 \\
193.64 \\
197.44 \\
183.72 \\
183.76\end{array}$ & $\begin{array}{l}122.71 \\
122.62 \\
122.60 \\
117.45 \\
122.61\end{array}$ \\
\hline $\begin{array}{l}299-E 27-1 \\
299-E 27-10 \\
299-E 27-11 \\
299-E 27-12 \\
299-E 27-13\end{array}$ & I & $\begin{array}{l}85.51 \\
67.81 \\
73.70 \\
79.00 \\
81.39\end{array}$ & $\begin{array}{l}208.04 \\
190.34 \\
196.07 \\
201.52 \\
203.97\end{array}$ & $\begin{array}{l}122.53 \\
122.53 \\
122.37 \\
122.52 \\
122.58\end{array}$ \\
\hline $\begin{array}{l}299-E 27-14 \\
299-E 27-15 \\
299-E 27-16 \\
299-E 27-17 \\
299-E 27-8\end{array}$ & & $\begin{array}{l}78.18 \\
76.77 \\
76.39 \\
70.99 \\
72.01\end{array}$ & $\begin{array}{l}200.72 \\
198.99 \\
198.77 \\
193.46 \\
194.41\end{array}$ & $\begin{array}{l}122.54 \\
122.22 \\
122.38 \\
122.47 \\
122.40\end{array}$ \\
\hline
\end{tabular}


Appendix A: June 1994 Water Level Measurement Data (Sheet 7 of 23)

\begin{tabular}{|c|c|c|c|c|}
\hline He11 & Notes & $\begin{array}{l}\text { Depth to } \\
\text { Water (m) }\end{array}$ & $\begin{array}{r}\text { Elevation, } \\
\text { Adjusted Casing }\end{array}$ & $\begin{array}{l}\text { m above ms } 1 \\
\text { Water Leve } 1\end{array}$ \\
\hline $\begin{array}{l}299-E 27-9 \\
299-E 28-12 \\
299-E 28-17 \\
299-E 28-18 \\
299-E 28-26\end{array}$ & $\begin{array}{l}\text { D } \\
\text { I }\end{array}$ & $\begin{array}{l}69.35 \\
93.36 \\
93.17 \\
88.60 \\
87.10\end{array}$ & $\begin{array}{l}191.78 \\
215.98 \\
215.97 \\
211.10 \\
209.48\end{array}$ & $\begin{array}{l}122.43 \\
122.62 \\
122.80 \\
122.50 \\
122.37\end{array}$ \\
\hline $\begin{array}{l}299-E 28-27 \\
299-E 28-28 \\
299-E 28-4 \\
299-E 28-6 \\
299-E 28-7\end{array}$ & I & $\begin{array}{l}85.01 \\
86.92 \\
88.23 \\
90.84 \\
86.80\end{array}$ & $\begin{array}{l}207.38 \\
209.26 \\
210.78 \\
213.39 \\
209.07\end{array}$ & $\begin{array}{l}122.36 \\
122.34 \\
122.55 \\
122.55 \\
122.26\end{array}$ \\
\hline $\begin{array}{l}299-E 28-9 \\
299-E 32-1 \\
299-E 32-10 \\
299-E 32-2 \\
299-E 32-3\end{array}$ & & $\begin{array}{l}91.07 \\
77.47 \\
72.03 \\
81.93 \\
83.87\end{array}$ & $\begin{array}{l}213.59 \\
200.00 \\
194.43 \\
204.23 \\
206.20\end{array}$ & $\begin{array}{l}122.52 \\
122.53 \\
122.39 \\
122.31 \\
122.33\end{array}$ \\
\hline $\begin{array}{l}299-E 32-4 \\
299-E 32-5 \\
299-E 32-6 \\
299-E 32-7 \\
299-E 32-8\end{array}$ & & $\begin{array}{l}86.77 \\
85.58 \\
81.02 \\
78.28 \\
74.40\end{array}$ & $\begin{array}{l}209.06 \\
207.92 \\
203.44 \\
200.69 \\
196.78\end{array}$ & $\begin{array}{l}122.29 \\
122.33 \\
122.41 \\
122.40 \\
122.38\end{array}$ \\
\hline $\begin{array}{l}299-E 32-9 \\
299-E 33-1 \\
299-E 33-12 \\
299-E 33-13 \\
299-E 33-14\end{array}$ & C & $\begin{array}{l}73.69 \\
70.16 \\
67.44 \\
69.30 \\
67.13\end{array}$ & $\begin{array}{l}196.09 \\
192.67 \\
190.03 \\
191.52 \\
189.60\end{array}$ & $\begin{array}{l}122.39 \\
122.50 \\
122.59 \\
122.22 \\
122.47\end{array}$ \\
\hline $\begin{array}{l}299-E 33-15 \\
299-E 33-17 \\
299-E 33-18 \\
299-E 33-2 \\
299-E 33-21\end{array}$ & & $\begin{array}{l}68.72 \\
70.05 \\
76.16 \\
70.10 \\
81.20\end{array}$ & $\begin{array}{l}191.20 \\
192.53 \\
198.69 \\
192.55 \\
203.71\end{array}$ & $\begin{array}{l}122.47 \\
122.48 \\
122.53 \\
122.45 \\
122.51\end{array}$ \\
\hline $\begin{array}{l}299-E 33-26 \\
299-E 33-28 \\
299-E 33-29 \\
299-E 33-3 \\
299-E 33-30\end{array}$ & D & $\begin{array}{l}70.75 \\
80.08 \\
83.00 \\
69.75 \\
79.92\end{array}$ & $\begin{array}{l}192.79 \\
202.46 \\
205.37 \\
192.20 \\
202.30\end{array}$ & $\begin{array}{l}122.04 \\
122.37 \\
122.36 \\
122.45 \\
122.37\end{array}$ \\
\hline
\end{tabular}


Appendix A: June 1994 Water Level Measurement Data (Sheet 8 of 23)

\begin{tabular}{|c|c|c|c|c|}
\hline We11 & Notes & $\begin{array}{l}\text { Depth to } \\
\text { Water }(m)\end{array}$ & $\begin{array}{r}\text { Elevation, } \\
\text { Adjusted Casing }\end{array}$ & $\begin{array}{l}\text { ve ms } 7 \\
\text { Water Leve] }\end{array}$ \\
\hline $\begin{array}{l}299-E 33-31 \\
299-E 33-32 \\
299-E 33-33 \\
299-E 33-34 \\
299-E 33-35\end{array}$ & & $\begin{array}{l}74.87 \\
78.83 \\
72.69 \\
70.70 \\
73.68\end{array}$ & $\begin{array}{l}197.35 \\
201.18 \\
195.18 \\
193.04 \\
195.99\end{array}$ & $\begin{array}{l}122.48 \\
122.34 \\
122.49 \\
122.34 \\
122.31\end{array}$ \\
\hline $\begin{array}{l}299-E 33-36 \\
299-E 33-37 \\
299-E 33-38 \\
299-E 33-39 \\
299-E 33-4\end{array}$ & & $\begin{array}{l}75.10 \\
76.62 \\
70.22 \\
67.64 \\
69.51\end{array}$ & $\begin{array}{l}197.11 \\
199.04 \\
192.62 \\
189.99 \\
191.96\end{array}$ & $\begin{array}{l}122.01 \\
122.42 \\
122.40 \\
122.35 \\
122.45\end{array}$ \\
\hline $\begin{array}{l}299-E 33-40 \\
299-E 33-41 \\
299-E 33-42 \\
299-E 33-43 \\
299-E 33-5\end{array}$ & $c$ & $\begin{array}{l}67.85 \\
77.18 \\
76.95 \\
79.54 \\
70.95\end{array}$ & $\begin{array}{l}190.37 \\
199.63 \\
199.43 \\
201.98 \\
193.46\end{array}$ & $\begin{array}{l}122.53 \\
122.45 \\
122.48 \\
122.44 \\
122.51\end{array}$ \\
\hline $\begin{array}{l}299-E 33-7 \\
299-E 33-8 \\
299-E 34-1 \\
299-E 34-10 \\
299-E 34-11\end{array}$ & & $\begin{array}{l}68.91 \\
75.89 \\
69.35 \\
72.52 \\
65.88\end{array}$ & $\begin{array}{l}191.34 \\
198.40 \\
191.86 \\
195.00 \\
188.35\end{array}$ & $\begin{array}{l}122.44 \\
122.51 \\
122.51 \\
122.48 \\
122.47\end{array}$ \\
\hline $\begin{array}{l}299-E 34-12 \\
299-E 34-2 \\
299-E 34-3 \\
299-E 34-5 \\
299-E 34-7\end{array}$ & & $\begin{array}{l}72.30 \\
69.83 \\
63.86 \\
57.34 \\
61.70\end{array}$ & $\begin{array}{l}194.72 \\
192.27 \\
186.39 \\
180.07 \\
184.18\end{array}$ & $\begin{array}{l}122.41 \\
122.44 \\
122.54 \\
122.73 \\
122.47\end{array}$ \\
\hline $\begin{array}{l}299-E 34-8 \\
299-E 34-9 \\
299-E 35-2 \\
299-W 10-10 \\
299-W 10-11\end{array}$ & I & $\begin{array}{l}72.92 \\
69.18 \\
60.90 \\
65.25 \\
65.22\end{array}$ & $\begin{array}{l}195.23 \\
191.62 \\
183.52 \\
205.85 \\
205.90\end{array}$ & $\begin{array}{l}122.31 \\
122.45 \\
122.62 \\
140.60 \\
140.68\end{array}$ \\
\hline $\begin{array}{l}299-W 10-12 \\
299-W 10-13 \\
299-W 10-14 \\
299-W 10-15 \\
299-W 10-16\end{array}$ & $\begin{array}{l}I \\
I\end{array}$ & $\begin{array}{l}65.13 \\
72.49 \\
72.73 \\
65.42 \\
64.43\end{array}$ & $\begin{array}{l}205.74 \\
213.07 \\
213.19 \\
206.01 \\
205.14\end{array}$ & $\begin{array}{l}140.61 \\
140.57 \\
140.46 \\
140.60 \\
140.70\end{array}$ \\
\hline
\end{tabular}


Appendix A: June 1994 Water Level Measurement Data (Sheet 9 of 23)

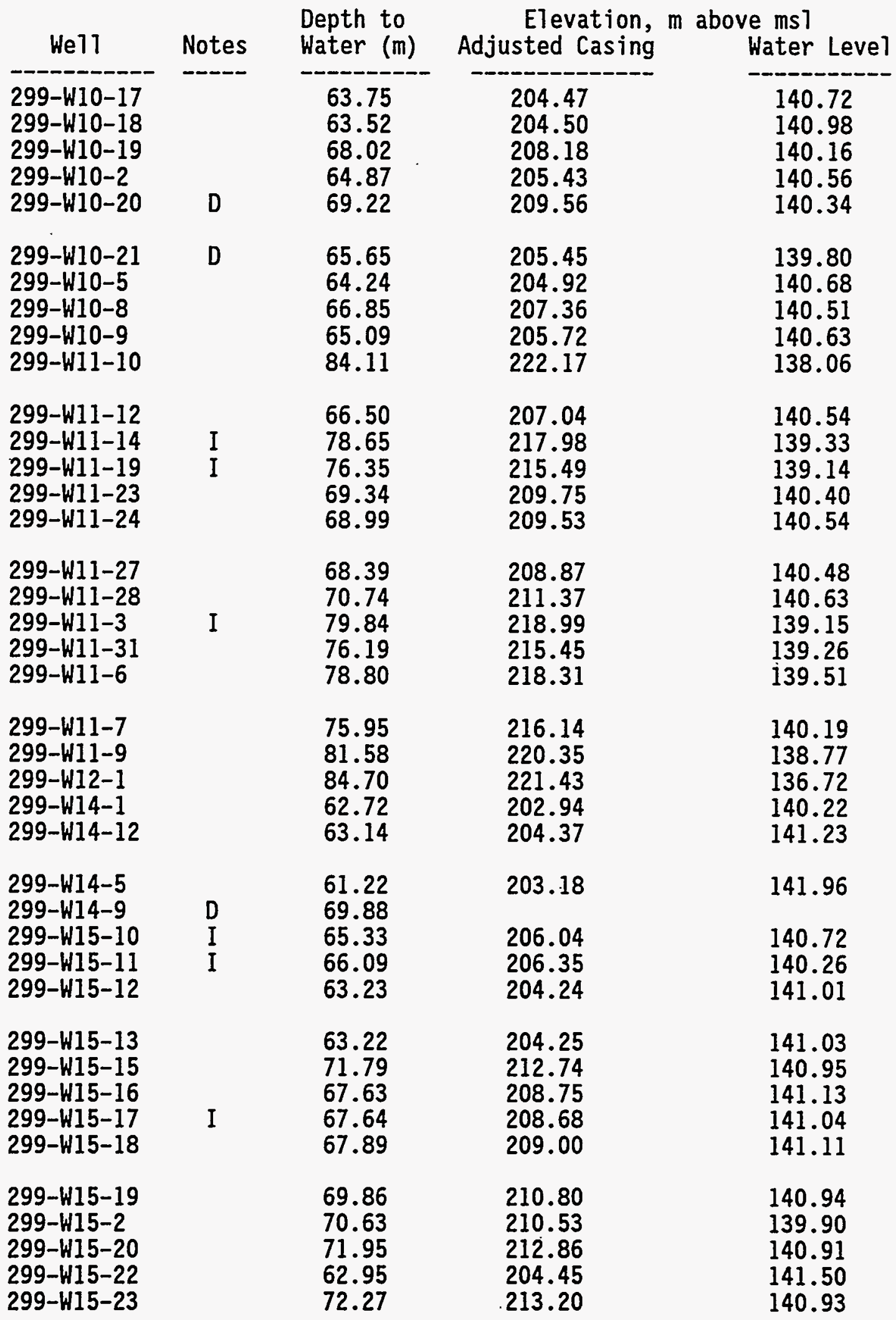


Appendix A: June 1994 Water Level Measurement Data (Sheet 10 of 23)

\begin{tabular}{|c|c|c|c|c|}
\hline Well & Notes & $\begin{array}{l}\text { Depth to } \\
\text { Water (m) }\end{array}$ & $\begin{array}{r}\text { Elevation, } \\
\text { Adjusted Casing }\end{array}$ & $\begin{array}{l}\text { m above ms } 1 \\
\text { Water Level }\end{array}$ \\
\hline $\begin{array}{l}299-W 15-24 \\
299-W 15-4 \\
299-W 15-5 \\
299-W 15-7 \\
299-W 18-15\end{array}$ & $\begin{array}{l}\text { I } \\
\text { I }\end{array}$ & $\begin{array}{l}72.22 \\
60.44 \\
62.87 \\
61.80 \\
60.37\end{array}$ & $\begin{array}{l}213.17 \\
201.78 \\
204.42 \\
202.39 \\
201.40\end{array}$ & $\begin{array}{l}140.95 \\
141.34 \\
141.56 \\
140.58 \\
141.03\end{array}$ \\
\hline $\begin{array}{l}299-W 18-17 \\
299-W 18-21 \\
299-W 18-22 \\
299-W 18-23 \\
299-W 18-24\end{array}$ & $\begin{array}{l}\text { D } \\
\text { I }\end{array}$ & $\begin{array}{l}30.96 \\
62.87 \\
62.97 \\
71.44 \\
67.45\end{array}$ & $\begin{array}{l}203.80 \\
203.76 \\
212.39 \\
208.59\end{array}$ & $\begin{array}{l}140.93 \\
140.78 \\
140.95 \\
141.14\end{array}$ \\
\hline $\begin{array}{l}299-W 18-25 \\
299-W 18-26 \\
299-W 18-28 \\
299-W 18-3 \\
299-W 18-30\end{array}$ & & $\begin{array}{l}61.78 \\
72.11 \\
66.25 \\
70.10 \\
63.75\end{array}$ & $\begin{array}{l}203.01 \\
213.07 \\
207.26 \\
209.70 \\
205.08\end{array}$ & $\begin{array}{l}141.23 \\
140.96 \\
141.02 \\
139.60 \\
141.33\end{array}$ \\
\hline $\begin{array}{l}299-W 18-31 \\
299-W 18-32 \\
299-W 18-33 \\
299-W 18-5 \\
299-W 19-12\end{array}$ & $\begin{array}{l}D \\
I\end{array}$ & $\begin{array}{l}61.16 \\
65.06 \\
62.83 \\
67.44 \\
63.84\end{array}$ & $\begin{array}{l}202.44 \\
206.24 \\
203.88 \\
208.18 \\
205.21\end{array}$ & $\begin{array}{l}141.27 \\
141.18 \\
141.06 \\
140.74 \\
141.37\end{array}$ \\
\hline $\begin{array}{l}299-W 19-14 \\
299-W 19-15 \\
299-W 19-2 \\
299-W 19-20 \\
299-W 19-21\end{array}$ & I & $\begin{array}{l}71.41 \\
71.16 \\
72.69 \\
72.44 \\
65.52\end{array}$ & $\begin{array}{l}211.29 \\
211.31 \\
211.54 \\
210.63 \\
206.90\end{array}$ & $\begin{array}{l}139.88 \\
140.15 \\
138.86 \\
138.19 \\
141.38\end{array}$ \\
\hline $\begin{array}{l}299-W 19-27 \\
299-W 19-28 \\
299-W 19-31 \\
299-W 19-32 \\
299-W 19-4\end{array}$ & I & $\begin{array}{l}67.13 \\
74.98 \\
64.13 \\
64.32 \\
79.08\end{array}$ & $\begin{array}{l}208.46 \\
214.38 \\
205.49 \\
205.71 \\
218.01\end{array}$ & $\begin{array}{l}141.33 \\
139.40 \\
141.36 \\
141.39 \\
138.93\end{array}$ \\
\hline $\begin{array}{l}299-W 19-6 \\
299-W 19-91 \\
299-W 19-92 \\
299-W 21-1 \\
299-W 22-19\end{array}$ & $\begin{array}{l}\text { C } \\
\text { I } \\
\text { I } \\
\text { I }\end{array}$ & $\begin{array}{l}69.27 \\
33.50 \\
33.32 \\
75.57 \\
69.44\end{array}$ & $\begin{array}{l}209.28 \\
206.62 \\
206.62 \\
213.13 \\
207.65\end{array}$ & $\begin{array}{l}140.00 \\
173.13 \\
173.31 \\
137.57 \\
138.21\end{array}$ \\
\hline
\end{tabular}


Appendix A: June 1994 Hater Level Measurement Data (Sheet 11 of 23)

\begin{tabular}{|c|c|c|c|c|}
\hline Well & Notes & $\begin{array}{l}\text { Depth to } \\
\text { Water. (m) }\end{array}$ & $\begin{array}{r}\text { Elevation, } \\
\text { Adjusted Casing }\end{array}$ & $\begin{array}{l}\text { ve ms1 } \\
\text { Water Level }\end{array}$ \\
\hline $\begin{array}{l}299-W 22-21 \\
299-W 22-22 \\
299-W 22-26 \\
299-W 22-39 \\
299-W 22-40\end{array}$ & $\begin{array}{l}\text { I } \\
\text { I } \\
\text { I }\end{array}$ & $\begin{array}{l}65.53 \\
71.67 \\
67.92 \\
64.24 \\
72.28\end{array}$ & $\begin{array}{l}204.22 \\
210.33 \\
207.36 \\
203.69 \\
210.99\end{array}$ & $\begin{array}{l}138.69 \\
138.66 \\
139.44 \\
139.44 \\
138.71\end{array}$ \\
\hline $\begin{array}{l}299-W 22-41 \\
299-W 22-42 \\
299-W 22-43 \\
299-W 22-44 \\
299-W 22-45\end{array}$ & & $\begin{array}{l}72.14 \\
71.98 \\
71.76 \\
66.63 \\
63.44\end{array}$ & $\begin{array}{l}210.84 \\
210.67 \\
210.72 \\
206.69 \\
203.06\end{array}$ & $\begin{array}{l}138.71 \\
138.68 \\
138.96 \\
140.06 \\
139.62\end{array}$ \\
\hline $\begin{array}{l}299-W 22-46 \\
299-W 22-7 \\
299-W 23-11 \\
299-W 23-13 \\
299-W 23-14\end{array}$ & I & $\begin{array}{l}65.22 \\
70.77 \\
62.23 \\
62.89 \\
62.35\end{array}$ & $\begin{array}{l}204.58 \\
209.52 \\
202.43 \\
203.10 \\
202.39\end{array}$ & $\begin{array}{l}139.36 \\
138.75 \\
140.20 \\
140.21 \\
140.03\end{array}$ \\
\hline $\begin{array}{l}299-W 23-15 \\
299-W 23-4 \\
299-W 23-6 \\
299-W 23-8 \\
299-W 26-10\end{array}$ & $\begin{array}{l}\text { I } \\
\text { I } \\
\text { I }\end{array}$ & $\begin{array}{l}60.14 \\
61.64 \\
63.83 \\
62.30 \\
65.49\end{array}$ & $\begin{array}{l}199.78 \\
201.97 \\
203.31 \\
202.37 \\
204.48\end{array}$ & $\begin{array}{l}139.64 \\
140.33 \\
139.48 \\
140.08 \\
138.99\end{array}$ \\
\hline \multirow{2}{*}{$\begin{array}{l}299-W 26-11 \\
299-W 26-12 \\
299-W 26-6 \\
299-W 26-7 \\
299-W 26-8\end{array}$} & $Q$ & \multirow{2}{*}{$\begin{array}{l}42.35 \\
66.86 \\
59.91 \\
59.19 \\
63.91\end{array}$} & $\begin{array}{l}205.56 \\
205.95\end{array}$ & $\begin{array}{l}163.21 \\
139.09\end{array}$ \\
\hline & & & $\begin{array}{l}198.73 \\
203.09\end{array}$ & $\begin{array}{l}139.54 \\
139.18\end{array}$ \\
\hline $\begin{array}{l}299-W 26-9 \\
299-W 27-2 \\
299-W 6-1 \\
299-W 6-10 \\
299-W 6-11\end{array}$ & I & $\begin{array}{l}60.29 \\
67.39 \\
75.45 \\
78.80 \\
75.85\end{array}$ & $\begin{array}{l}199.39 \\
206.38 \\
214.13 \\
217.16 \\
214.23\end{array}$ & $\begin{array}{l}139.10 \\
138.99 \\
138.68 \\
138.36 \\
138.38\end{array}$ \\
\hline $\begin{array}{l}299-W 6-12 \\
299-W 6-2 \\
299-W 6-3 \\
299-W 6-4 \\
299-W 6-6\end{array}$ & $\begin{array}{l}\text { I } \\
\text { I }\end{array}$ & $\begin{array}{l}72.36 \\
71.80 \\
74.27 \\
74.49 \\
78.29\end{array}$ & $\begin{array}{l}211.08 \\
211.06 \\
213.31 \\
213.74 \\
216.41\end{array}$ & $\begin{array}{l}138.72 \\
139.25 \\
139.03 \\
139.25 \\
138.12\end{array}$ \\
\hline
\end{tabular}


Appendix A: June 1994 Water Level Measurement Data (Sheet 12 of 23)

\begin{tabular}{|c|c|c|c|c|}
\hline We11 & Notes & $\begin{array}{l}\text { Depth to } \\
\text { Water (m) }\end{array}$ & $\begin{array}{r}\text { Elevation, } \\
\text { Adjusted Casing }\end{array}$ & $\begin{array}{l}\text { ve ms } 1 \\
\text { Water Leve1 }\end{array}$ \\
\hline $\begin{array}{l}299-W 6-7 \\
299-W 6-9 \\
299-W 7-1 \\
299-W 7-10 \\
299-W 7-11\end{array}$ & & $\begin{array}{l}78.35 \\
72.97 \\
71.09 \\
71.16 \\
68.35\end{array}$ & $\begin{array}{l}216.49 \\
212.14 \\
210.53 \\
210.21 \\
207.71\end{array}$ & $\begin{array}{l}138.14 \\
139.17 \\
139.44 \\
139.05 \\
139.36\end{array}$ \\
\hline $\begin{array}{l}299-W 7-12 \\
299-W 7-2 \\
299-W 7-3 \\
299-W 7-4 \\
299-W 7-5\end{array}$ & I & $\begin{array}{l}70.25 \\
66.92 \\
67.30 \\
65.06 \\
66.17\end{array}$ & $\begin{array}{l}209.68 \\
205.92 \\
206.09 \\
204.73 \\
205.15\end{array}$ & $\begin{array}{l}139.43 \\
139.00 \\
138.79 \\
139.67 \\
138.97\end{array}$ \\
\hline $\begin{array}{l}299-W 7-6 \\
299-W 7-7 \\
299-W 7-8 \\
299-W 7-9 \\
299-W 8-1\end{array}$ & & $\begin{array}{l}67.92 \\
66.73 \\
70.94 \\
71.37 \\
74.18\end{array}$ & $\begin{array}{l}206.85 \\
205.72 \\
209.50 \\
210.95 \\
213.77\end{array}$ & $\begin{array}{l}138.93 \\
138.99 \\
138.57 \\
139.58 \\
139.59\end{array}$ \\
\hline $\begin{array}{l}299-W 9-1 \\
3099-47-18 B \\
399-1-1 \\
399-1-10 A \\
399-1-11\end{array}$ & & $\begin{array}{r}84.56 \\
9.63 \\
9.96 \\
9.01 \\
10.23\end{array}$ & $\begin{array}{l}224.86 \\
114.28 \\
114.81 \\
113.86 \\
115.13\end{array}$ & $\begin{array}{l}140.30 \\
104.65 \\
104.85 \\
104.85 \\
104.90\end{array}$ \\
\hline $\begin{array}{l}399-1-12 \\
399-1-13 A \\
399-1-14 A \\
399-1-15 \\
399-1-16 A\end{array}$ & D & $\begin{array}{l}12.37 \\
13.62 \\
11.87 \\
10.71 \\
11.55\end{array}$ & $\begin{array}{l}117.17 \\
118.45 \\
116.79 \\
115.68 \\
116.27\end{array}$ & $\begin{array}{l}104.80 \\
104.84 \\
104.92 \\
104.97 \\
104.72\end{array}$ \\
\hline $\begin{array}{l}399-1-16 B \\
399-1-16 C \\
399-1-17 A \\
399-1-17 B \\
399-1-17 C\end{array}$ & $\begin{array}{l}\text { I } \\
\text { I } \\
\text { I } \\
\text { I }\end{array}$ & $\begin{array}{r}11.38 \\
2.38 \\
10.28 \\
10.66 \\
.42\end{array}$ & $\begin{array}{l}116.15 \\
116.50 \\
115.04 \\
115.15 \\
115.23\end{array}$ & $\begin{array}{l}104.77 \\
114.12 \\
104.76 \\
104.49 \\
114.82\end{array}$ \\
\hline $\begin{array}{l}399-1-18 A \\
399-1-18 B \\
399-1-18 C \\
399-1-19 \\
399-1-2\end{array}$ & $\begin{array}{l}\text { I } \\
\text { I } \\
\text { D }\end{array}$ & $\begin{array}{r}14.02 \\
13.67 \\
12.93 \\
9.36 \\
13.19\end{array}$ & $\begin{array}{l}119.11 \\
118.84 \\
118.27 \\
114.19 \\
117.20\end{array}$ & $\begin{array}{l}105.09 \\
105.17 \\
105.34 \\
104.83 \\
104.01\end{array}$ \\
\hline
\end{tabular}


Appendix A: June 1994 Hater Level Measurement Data (Sheet 13 of 23)

\begin{tabular}{|c|c|c|c|c|}
\hline He11 & Notes & $\begin{array}{l}\text { Depth to } \\
\text { Water (m) }\end{array}$ & $\begin{array}{r}\text { Elevation, } \\
\text { Adjusted Casing }\end{array}$ & $\begin{array}{l}\text { e ms } 1 \\
\text { Water Leve1 }\end{array}$ \\
\hline $\begin{array}{l}399-1-21 A \\
399-1-21 B \\
399-1-3 \\
399-1-4 \\
399-1-5\end{array}$ & $\begin{array}{l}D \\
D\end{array}$ & $\begin{array}{l}11.81 \\
12.10 \\
12.52 \\
11.09 \\
10.95\end{array}$ & $\begin{array}{l}116.55 \\
116.84 \\
117.26 \\
116.00 \\
115.75\end{array}$ & $\begin{array}{l}104.74 \\
104.74 \\
104.74 \\
104.92 \\
104.81\end{array}$ \\
\hline $\begin{array}{l}399-1-6 \\
399-1-7 \\
399-1-8 \\
399-1-9 \\
399-2-1\end{array}$ & $\begin{array}{l}\text { I } \\
\text { I }\end{array}$ & $\begin{array}{r}8.99 \\
12.81 \\
12.58 \\
6.97 \\
9.69\end{array}$ & $\begin{array}{l}113.94 \\
117.53 \\
117.31 \\
117.27 \\
114.37\end{array}$ & $\begin{array}{l}104.95 \\
104.72 \\
104.73 \\
110.31 \\
104.68\end{array}$ \\
\hline $\begin{array}{l}399-2-2 \\
399-2-3 \\
399-3-1 \\
399-3-10 \\
399-3-12\end{array}$ & & $\begin{array}{r}10.33 \\
9.69 \\
12.52 \\
12.85 \\
13.73\end{array}$ & $\begin{array}{l}115.07 \\
114.43 \\
117.16 \\
117.45 \\
118.27\end{array}$ & $\begin{array}{l}104.74 \\
104.74 \\
104.65 \\
104.60 \\
104.55\end{array}$ \\
\hline $\begin{array}{l}399-3-6 \\
399-3-9 \\
399-4-1 \\
399-4-10 \\
399-4-11\end{array}$ & I & $\begin{array}{l}15.02 \\
13.65 \\
15.99 \\
10.81 \\
18.64\end{array}$ & $\begin{array}{l}119.73 \\
118.29 \\
120.58 \\
115.38 \\
123.28\end{array}$ & $\begin{array}{l}104.70 \\
104.64 \\
104.59 \\
104.57 \\
104.63\end{array}$ \\
\hline $\begin{array}{l}399-4-7 \\
399-4-9 \\
399-5-1 \\
399-6-1 \\
399-8-1\end{array}$ & $\begin{array}{l}\text { I } \\
\text { I }\end{array}$ & $\begin{array}{l}10.84 \\
11.88 \\
15.82 \\
13.69 \\
15.93\end{array}$ & $\begin{array}{l}115.39 \\
116.48 \\
120.56 \\
118.50 \\
120.74\end{array}$ & $\begin{array}{l}104.55 \\
104.61 \\
104.74 \\
104.81 \\
104.81\end{array}$ \\
\hline $\begin{array}{l}399-8-2 \\
399-8-3 \\
399-8-5 A \\
399-8-5 B \\
399-8-5 C\end{array}$ & $\begin{array}{l}\text { I } \\
\text { I } \\
D \\
I \\
D\end{array}$ & $\begin{array}{r}16.34 \\
15.41 \\
17.04 \\
16.79 \\
8.89\end{array}$ & $\begin{array}{l}121.32 \\
120.32 \\
122.01 \\
121.87 \\
121.89\end{array}$ & $\begin{array}{l}104.98 \\
104.91 \\
104.97 \\
105.08 \\
113.00\end{array}$ \\
\hline $\begin{array}{l}699-10-54 A \\
699-10-E 12 \\
699-101-48 B \\
699-11-45 A \\
699-14-38\end{array}$ & & $\begin{array}{r}31.44 \\
22.53 \\
2.69 \\
50.81 \\
33.65\end{array}$ & $\begin{array}{l}157.40 \\
131.33 \\
118.92 \\
176.35 \\
156.94\end{array}$ & $\begin{array}{l}125.96 \\
108.79 \\
116.23 \\
125.54 \\
123.29\end{array}$ \\
\hline
\end{tabular}


Appendix A: June 1994 Water Level Measurement Data (Sheet 14 of 23)

\begin{tabular}{|c|c|c|c|c|}
\hline Well & Notes & $\begin{array}{l}\text { Depth to } \\
\text { Water (m) }\end{array}$ & $\begin{array}{r}\text { Elevation, } \\
\text { Adjusted Casing }\end{array}$ & $\begin{array}{l}\text { m above ms } 1 \\
\text { Water Leve } 1\end{array}$ \\
\hline $\begin{array}{l}699-14-47 \\
699-15-15 A \\
699-15-26 \\
699-17-5 \\
699-17-70\end{array}$ & & $\begin{array}{l}53.43 \\
42.67 \\
37.88 \\
13.99 \\
27.05\end{array}$ & $\begin{array}{l}178.99 \\
166.77 \\
159.66 \\
132.04 \\
171.66\end{array}$ & $\begin{array}{l}125.55 \\
124.10 \\
121.78 \\
118.05 \\
144.61\end{array}$ \\
\hline $\begin{array}{l}699-19-43 \\
699-19-58 \\
699-19-88 \\
699-2-3 \\
699-2-33 A\end{array}$ & & $\begin{array}{l}45.45 \\
47.19 \\
39.81 \\
26.65 \\
40.30\end{array}$ & $\begin{array}{l}168.12 \\
174.67 \\
196.43 \\
145.43 \\
163.49\end{array}$ & $\begin{array}{l}122.67 \\
127.47 \\
156.62 \\
118.78 \\
123.19\end{array}$ \\
\hline $\begin{array}{l}699-20-20 \\
699-20-39 \\
699-20-41 P \\
699-20-41 Q \\
699-20-41 R\end{array}$ & $\begin{array}{l}\text { I } \\
\text { D } \\
\text { D } \\
\text { D }\end{array}$ & $\begin{array}{l}32.33 \\
42.24 \\
40.97 \\
41.45 \\
42.18\end{array}$ & $\begin{array}{l}154.10 \\
164.59 \\
162.15 \\
162.15 \\
162.15\end{array}$ & $\begin{array}{l}121.77 \\
122.34 \\
121.19 \\
120.71 \\
119.98\end{array}$ \\
\hline $\begin{array}{l}699-20-E 12 \\
699-21-17 \\
699-23-34 A \\
699-24-11 \\
699-24-33\end{array}$ & $\begin{array}{l}D \\
D \\
C\end{array}$ & $\begin{array}{l}24.41 \\
40.13 \\
40.15 \\
30.88 \\
37.53\end{array}$ & $\begin{array}{l}133.27 \\
160.72 \\
162.42 \\
144.94 \\
159.78\end{array}$ & $\begin{array}{l}108.87 \\
120.59 \\
122.26 \\
114.07 \\
122.25\end{array}$ \\
\hline $\begin{array}{l}699-24-34 A \\
699-24-34 B \\
699-24-34 C \\
699-24-35 \\
699-25-33 A\end{array}$ & I & $\begin{array}{l}40.46 \\
40.38 \\
40.06 \\
41.95 \\
38.98\end{array}$ & $\begin{array}{l}162.73 \\
162.61 \\
162.33 \\
164.23 \\
161.23\end{array}$ & $\begin{array}{l}122.27 \\
122.23 \\
122.27 \\
122.28 \\
122.25\end{array}$ \\
\hline $\begin{array}{l}699-25-34 A \\
699-25-34 B \\
699-25-34 C \\
699-25-34 D \\
699-25-55\end{array}$ & & $\begin{array}{l}39.40 \\
39.25 \\
40.93 \\
41.67 \\
80.58\end{array}$ & $\begin{array}{l}161.64 \\
161.36 \\
163.21 \\
163.95 \\
206.21\end{array}$ & $\begin{array}{l}122.24 \\
122.11 \\
122.27 \\
122.29 \\
125.64\end{array}$ \\
\hline $\begin{array}{l}699-25-70 \\
699-26-15 A \\
699-26-33 \\
699-26-34 A \\
699-26-34 B\end{array}$ & & $\begin{array}{l}56.21 \\
13.92 \\
41.09 \\
38.93 \\
39.34\end{array}$ & $\begin{array}{l}191.96 \\
134.92 \\
163.22 \\
161.06 \\
161.63\end{array}$ & $\begin{array}{l}135.75 \\
120.99 \\
122.12 \\
122.12 \\
122.29\end{array}$ \\
\hline
\end{tabular}


Appendix A: June 1994 Water Leve1 Measurement Data (Sheet 15 of 23)

\begin{tabular}{|c|c|c|c|c|}
\hline Hell & Notes & $\begin{array}{l}\text { Depth to } \\
\text { Water (m) }\end{array}$ & $\begin{array}{l}\text { Elevation, } \\
\text { Adjusted Casing }\end{array}$ & $\begin{array}{l}\text { m above ms } 1 \\
\text { Water Level }\end{array}$ \\
\hline $\begin{array}{l}699-26-35 A \\
699-26-35 C \\
699-26-83 B P \\
699-26-83 B Q \\
699-26-83 B R\end{array}$ & $\begin{array}{l}I \\
D \\
D \\
D\end{array}$ & $\begin{array}{l}40.23 \\
40.08 \\
71.23 \\
71.13 \\
72.37\end{array}$ & $\begin{array}{l}162.35 \\
162.36 \\
193.85 \\
193.85 \\
193.85\end{array}$ & $\begin{array}{l}122.12 \\
122.28 \\
122.62 \\
122.72 \\
121.49\end{array}$ \\
\hline $\begin{array}{l}699-26-89 \\
699-27-8 \\
699-28-40 \\
699-28-52 A \\
699-29-70 A P\end{array}$ & $\begin{array}{l}I \\
C\end{array}$ & $\begin{array}{l}55.60 \\
22.29 \\
48.14 \\
85.76 \\
58.31\end{array}$ & $\begin{array}{l}199.06 \\
141.94 \\
170.52 \\
208.69 \\
191.95\end{array}$ & $\begin{array}{l}143.46 \\
119.65 \\
122.38 \\
122.93 \\
133.64\end{array}$ \\
\hline $\begin{array}{l}699-29-70 A Q \\
699-29-70 C P \\
699-29-70 C Q \\
699-29-70 C R \\
699-29-70 C S\end{array}$ & $\begin{array}{l}c \\
C \\
C \\
C \\
C\end{array}$ & $\begin{array}{l}56.42 \\
70.19 \\
71.03 \\
70.53 \\
71.29\end{array}$ & $\begin{array}{l}192.07 \\
192.31 \\
192.33 \\
192.37 \\
192.42\end{array}$ & $\begin{array}{l}135.65 \\
122.12 \\
121.30 \\
121.84 \\
121.13\end{array}$ \\
\hline $\begin{array}{l}699-29-70 C T \\
699-29-70 C U \\
699-29-70 D P \\
699-29-78 \\
699-3-45\end{array}$ & $\begin{array}{l}C \\
C \\
C\end{array}$ & $\begin{array}{l}71.56 \\
71.90 \\
63.62 \\
57.37 \\
28.31\end{array}$ & $\begin{array}{l}192.48 \\
192.52 \\
192.76 \\
197.22 \\
153.78\end{array}$ & $\begin{array}{l}120.92 \\
120.63 \\
129.14 \\
139.85 \\
125.47\end{array}$ \\
\hline $\begin{array}{l}699-31-31 \\
699-31-65 \\
699-31-84 B \\
699-32-22 A \\
699-32-43\end{array}$ & $\begin{array}{l}I \\
I \\
C \\
D\end{array}$ & $\begin{array}{l}39.08 \\
74.50 \\
62.35 \\
36.10 \\
35.03\end{array}$ & $\begin{array}{l}161.34 \\
208.21 \\
190.54 \\
157.47\end{array}$ & $\begin{array}{l}122.26 \\
133.70 \\
128.19 \\
122.43\end{array}$ \\
\hline $\begin{array}{l}699-32-62 \\
699-32-70 B \\
699-32-72 \\
699-32-72 B \\
699-32-77\end{array}$ & $\begin{array}{l}I \\
I \\
D \\
I\end{array}$ & $\begin{array}{l}84.93 \\
66.63 \\
66.54 \\
66.96 \\
59.91\end{array}$ & $\begin{array}{l}215.52 \\
203.20 \\
203.66 \\
199.26\end{array}$ & $\begin{array}{l}130.59 \\
136.57 \\
137.11 \\
139.35\end{array}$ \\
\hline $\begin{array}{l}699-33-42 \\
699-33-56 \\
699-34-39 A \\
699-34-41 B \\
699-34-42\end{array}$ & I & $\begin{array}{l}35.05 \\
95.78 \\
41.29 \\
51.56 \\
42.19\end{array}$ & $\begin{array}{l}157.28 \\
218.55 \\
163.70 \\
174.01 \\
164.65\end{array}$ & $\begin{array}{l}122.23 \\
122.77 \\
122.41 \\
122.45 \\
122.46\end{array}$ \\
\hline $\begin{array}{l}699-34-51 \\
699-34-61 \\
699-34-88 \\
699-35-66 \\
699-35-70\end{array}$ & $D$ & $\begin{array}{r}101.92 \\
93.55 \\
50.54 \\
87.94 \\
74.89\end{array}$ & $\begin{array}{l}224.56 \\
220.77 \\
192.88 \\
221.18 \\
211.45\end{array}$ & $\begin{array}{l}122.65 \\
127.22 \\
142.34 \\
133.24 \\
136.55\end{array}$ \\
\hline
\end{tabular}


Appendix A: June 1994 Water Leve1 Measurement Data (Sheet 16 of 23)

\begin{tabular}{|c|c|c|c|c|}
\hline Well & Notes & $\begin{array}{l}\text { Depth to } \\
\text { Water (m) }\end{array}$ & $\begin{array}{r}\text { Elevation, } \\
\text { Adjusted Casing }\end{array}$ & $\begin{array}{l}\text { m above ms } 1 \\
\quad \text { Water Leve] }\end{array}$ \\
\hline $\begin{array}{l}699-35-78 A \\
699-35-9 \\
699-36-61 A \\
699-36-93 \\
699-37-43\end{array}$ & I & $\begin{array}{r}60.78 \\
35.26 \\
103.94 \\
53.28 \\
87.61\end{array}$ & $\begin{array}{l}201.37 \\
152.35 \\
228.02 \\
196.53 \\
210.36\end{array}$ & $\begin{array}{l}140.58 \\
117.09 \\
124.08 \\
143.25 \\
122.76\end{array}$ \\
\hline $\begin{array}{l}699-37-82 A \\
699-38-61 \\
699-38-65 \\
699-38-70 \\
699-39-39\end{array}$ & $\begin{array}{l}\text { I } \\
\text { D }\end{array}$ & $\begin{array}{r}52.79 \\
103.53 \\
99.01 \\
79.62 \\
37.65\end{array}$ & $\begin{array}{l}194.08 \\
227.13 \\
229.61 \\
216.61 \\
163.57\end{array}$ & $\begin{array}{l}141.29 \\
123.61 \\
130.61 \\
136.99 \\
125.92\end{array}$ \\
\hline $\begin{array}{l}699-39-79 \\
699-40-1 \\
699-40-33 A \\
699-40-36 \\
699-40-39\end{array}$ & $\begin{array}{l}c \\
c\end{array}$ & $\begin{array}{l}64.30 \\
23.06 \\
32.82 \\
35.97 \\
39.34\end{array}$ & $\begin{array}{l}205.29 \\
133.72 \\
157.90 \\
161.21 \\
165.15\end{array}$ & $\begin{array}{l}140.99 \\
110.65 \\
125.08 \\
125.25 \\
125.82\end{array}$ \\
\hline $\begin{array}{l}699-40-40 A \\
699-40-40 B \\
699-40-62 \\
699-41-23 \\
699-41-35\end{array}$ & $\begin{array}{l}c \\
c\end{array}$ & $\begin{array}{r}39.46 \\
39.67 \\
104.65 \\
21.33 \\
33.08\end{array}$ & $\begin{array}{l}164.96 \\
165.26 \\
227.92 \\
142.19 \\
158.61\end{array}$ & $\begin{array}{l}125.50 \\
125.59 \\
123.27 \\
120.86 \\
125.53\end{array}$ \\
\hline $\begin{array}{l}699-41-40 \\
699-41-42 \\
699-42-37 \\
699-42-39 A \\
699-42-39 B\end{array}$ & $\begin{array}{l}c \\
c\end{array}$ & $\begin{array}{l}39.58 \\
70.75 \\
31.83 \\
42.10 \\
42.19\end{array}$ & $\begin{array}{l}166.40 \\
196.26 \\
158.32 \\
170.12 \\
170.18\end{array}$ & $\begin{array}{l}126.82 \\
125.51 \\
126.49 \\
128.02 \\
127.98\end{array}$ \\
\hline $\begin{array}{l}699-42-40 A \\
699-42-40 B \\
699-42-40 C \\
699-42-41 \\
699-42-42 B\end{array}$ & $\begin{array}{l}C \\
C \\
D\end{array}$ & $\begin{array}{l}37.54 \\
37.52 \\
40.39 \\
44.62 \\
50.70\end{array}$ & $\begin{array}{l}166.28 \\
166.56 \\
166.47 \\
172.91 \\
177.77\end{array}$ & $\begin{array}{l}128.74 \\
129.04 \\
126.08 \\
128.29 \\
127.07\end{array}$ \\
\hline $\begin{array}{l}699-43-104 \\
699-43-40 \\
699-43-41 E \\
699-43-41 F \\
699-43-41 G\end{array}$ & $\begin{array}{l}c \\
c \\
c\end{array}$ & $\begin{array}{l}83.25 \\
36.77 \\
39.44 \\
39.47 \\
40.76\end{array}$ & $\begin{array}{l}233.50 \\
165.26 \\
167.90 \\
167.95 \\
168.05\end{array}$ & $\begin{array}{l}150.25 \\
128.49 \\
128.46 \\
128.48 \\
127.28\end{array}$ \\
\hline
\end{tabular}


Appendix A: June 1994 Water Level Measurement Data (Sheet 17 of 23)

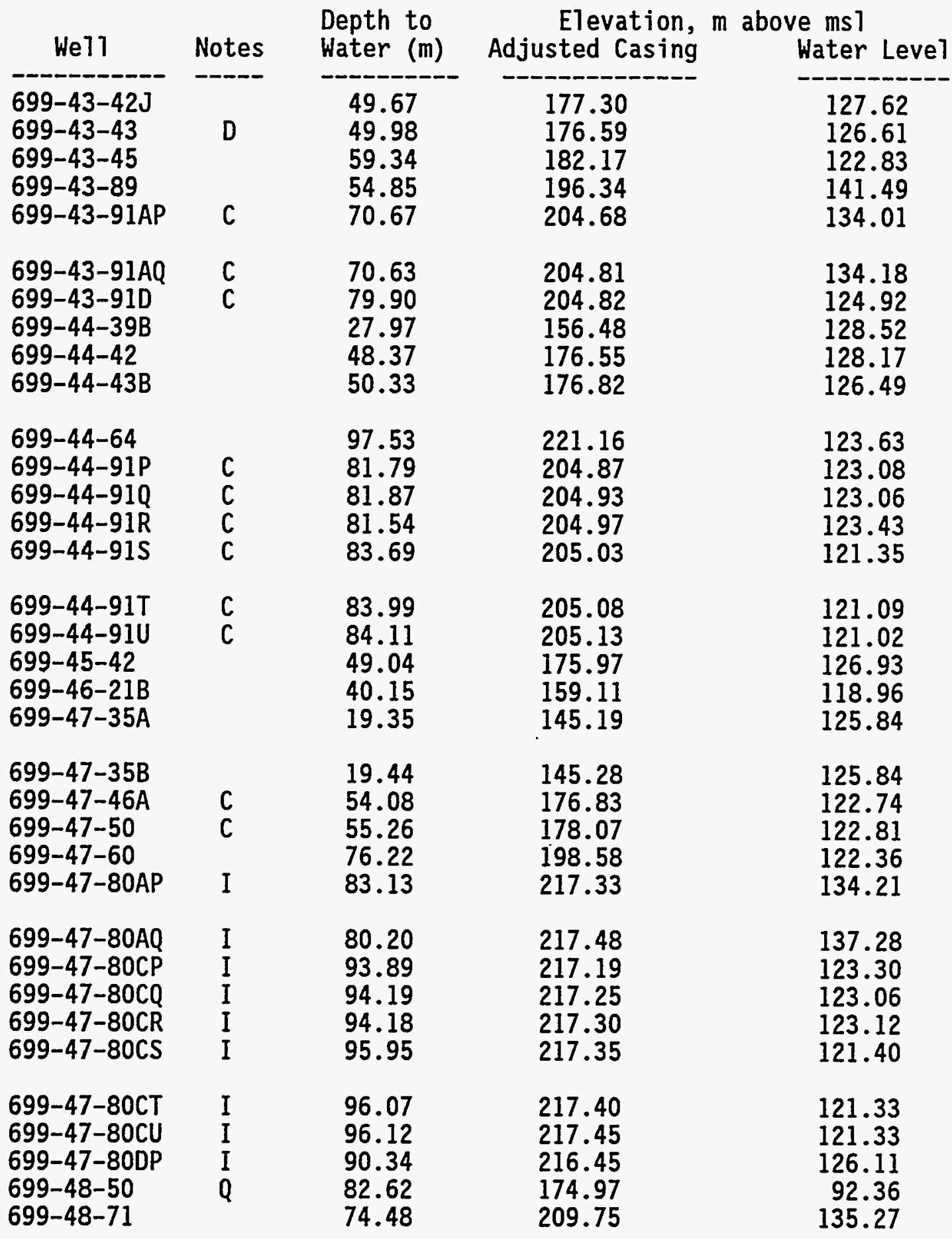




\section{WHC-EP-0394-9}

Appendix A: June 1994 Water Level Measurement Data (Sheet 18 of 23)

\begin{tabular}{|c|c|c|c|c|}
\hline Well & Notes & $\begin{array}{l}\text { Depth to } \\
\text { Water }(\mathrm{m})\end{array}$ & $\begin{array}{r}\text { Elevation, } \\
\text { Adjusted Casing }\end{array}$ & $\begin{array}{l}\text { m above ms } 1 \\
\text { Water Level }\end{array}$ \\
\hline $\begin{array}{l}699-48-7 A \\
699-49-111 \\
699-49-13 E \\
699-49-28 \\
699-49-55 A\end{array}$ & $\begin{array}{l}D \\
D\end{array}$ & $\begin{array}{r}7.94 \\
13.22 \\
15.73 \\
43.34 \\
39.46\end{array}$ & $\begin{array}{l}278.32 \\
125.80 \\
163.19 \\
161.86\end{array}$ & $\begin{array}{l}265.10 \\
110.07 \\
119.85 \\
122.40\end{array}$ \\
\hline $\begin{array}{l}699-49-55 B \\
699-49-57 A \\
699-49-57 B \\
699-49-79 \\
699-50-28 B\end{array}$ & $\begin{array}{l}c \\
c\end{array}$ & $\begin{array}{l}39.48 \\
46.33 \\
47.11 \\
71.58 \\
44.02\end{array}$ & $\begin{array}{l}161.89 \\
168.71 \\
169.47 \\
210.07 \\
163.77\end{array}$ & $\begin{array}{l}122.40 \\
122.38 \\
122.36 \\
138.49 \\
119.75\end{array}$ \\
\hline $\begin{array}{l}699-50-30 \\
699-50-42 \\
699-50-45 \\
699-50-48 B \\
699-50-53 A\end{array}$ & $\begin{array}{l}I \\
C \\
I\end{array}$ & $\begin{array}{l}41.21 \\
17.34 \\
13.39 \\
44.33 \\
47.56\end{array}$ & $\begin{array}{l}161.19 \\
142.29 \\
137.59 \\
167.76 \\
169.91\end{array}$ & $\begin{array}{l}119.98 \\
124.96 \\
124.20 \\
123.43 \\
122.36\end{array}$ \\
\hline $\begin{array}{l}699-50-53 B \\
699-50-85 \\
699-51-46 \\
699-51-63 \\
699-51-75\end{array}$ & $\begin{array}{l}c \\
c\end{array}$ & $\begin{array}{l}47.51 \\
87.01 \\
11.80 \\
51.23 \\
59.07\end{array}$ & $\begin{array}{l}169.96 \\
225.35 \\
135.52 \\
174.30 \\
195.53\end{array}$ & $\begin{array}{l}122.45 \\
138.35 \\
123.73 \\
123.06 \\
136.46\end{array}$ \\
\hline $\begin{array}{l}699-52-19 \\
699-52-46 A \\
699-52-48 \\
699-52-54 \\
699-52-57\end{array}$ & $\begin{array}{l}c \\
c\end{array}$ & $\begin{array}{l}15.26 \\
14.65 \\
18.99 \\
51.01 \\
49.18\end{array}$ & $\begin{array}{l}125.30 \\
138.87 \\
142.06 \\
173.26 \\
171.24\end{array}$ & $\begin{array}{l}110.04 \\
124.22 \\
123.06 \\
122.26 \\
122.05\end{array}$ \\
\hline $\begin{array}{l}699-53-111 \\
699-53-114 \\
699-53-35 \\
699-53-47 A \\
699-53-47 B\end{array}$ & $\begin{array}{l}D \\
D \\
D\end{array}$ & $\begin{array}{l}17.24 \\
31.96 \\
39.79 \\
10.10 \\
10.20\end{array}$ & $\begin{array}{l}282.67 \\
297.21 \\
161.85 \\
133.59 \\
133.68\end{array}$ & $\begin{array}{l}265.44 \\
265.25 \\
122.05 \\
123.48 \\
123.48\end{array}$ \\
\hline $\begin{array}{l}699-53-48 A \\
699-53-48 B \\
699-53-50 \\
699-53-55 A \\
699-53-55 B\end{array}$ & $\begin{array}{l}D \\
C \\
I \\
I\end{array}$ & $\begin{array}{l}12.09 \\
11.53 \\
12.62 \\
53.56 \\
53.73\end{array}$ & $\begin{array}{l}134.86 \\
134.94 \\
135.40 \\
175.74 \\
175.82\end{array}$ & $\begin{array}{l}122.77 \\
123.41 \\
122.78 \\
122.17 \\
122.09\end{array}$ \\
\hline
\end{tabular}


Appendix A: June 1994 Water Leve1 Measurement Data (Sheet 19 of 23)

\begin{tabular}{|c|c|c|c|c|}
\hline Well & Notes & $\begin{array}{l}\text { Depth to } \\
\text { Water (m) }\end{array}$ & $\begin{array}{l}\text { Elevation, } \\
\text { Adjusted Casing }\end{array}$ & $\begin{array}{l}\text { m above ms } 1 \\
\text { Water Level }\end{array}$ \\
\hline $\begin{array}{l}699-53-55 C \\
699-54-19 \\
699-54-34 \\
699-54-42 \\
699-54-45 A\end{array}$ & I & $\begin{array}{r}53.43 \\
6.86 \\
42.96 \\
35.12 \\
29.40\end{array}$ & $\begin{array}{l}175.60 \\
116.92 \\
167.71 \\
155.90 \\
150.66\end{array}$ & $\begin{array}{l}122.18 \\
110.06 \\
124.76 \\
120.79 \\
121.26\end{array}$ \\
\hline $\begin{array}{l}699-54-48 \\
699-54-57 \\
699-55-21 \\
699-55-44 \\
699-55-50 c\end{array}$ & $\begin{array}{l}C \\
D\end{array}$ & $\begin{array}{l}17.13 \\
53.41 \\
11.07 \\
37.73 \\
13.27\end{array}$ & $\begin{array}{l}139.30 \\
175.64 \\
120.69 \\
158.40 \\
135.46\end{array}$ & $\begin{array}{l}122.17 \\
122.23 \\
109.62 \\
120.67 \\
122.19\end{array}$ \\
\hline $\begin{array}{l}699-55-55 \\
699-55-57 \\
699-55-70 \\
699-55-76 \\
699-55-89\end{array}$ & I & $\begin{array}{l}49.70 \\
51.01 \\
42.31 \\
42.81 \\
49.90\end{array}$ & $\begin{array}{l}171.48 \\
173.19 \\
173.44 \\
177.77 \\
188.19\end{array}$ & $\begin{array}{l}121.78 \\
122.18 \\
131.13 \\
134.97 \\
138.29\end{array}$ \\
\hline $\begin{array}{l}699-55-95 \\
699-56-43 \\
699-56-53 \\
699-57-25 A \\
699-57-29 A\end{array}$ & $\begin{array}{l}I \\
C \\
C \\
N \\
N\end{array}$ & $\begin{array}{l}95.29 \\
40.45 \\
10.11 \\
15.60 \\
16.57\end{array}$ & $\begin{array}{l}236.84 \\
164.72 \\
132.39 \\
126.36 \\
124.50\end{array}$ & $\begin{array}{l}141.56 \\
124.27 \\
122.27 \\
110.76 \\
107.93\end{array}$ \\
\hline $\begin{array}{l}699-57-29 B \\
699-57-59 \\
699-57-83 A \\
699-57-83 B P \\
699-57-83 B Q\end{array}$ & $\begin{array}{l}c \\
c\end{array}$ & $\begin{array}{l}16.77 \\
53.46 \\
44.47 \\
54.63 \\
54.73\end{array}$ & $\begin{array}{l}126.85 \\
175.64 \\
176.16 \\
176.35 \\
176.40\end{array}$ & $\begin{array}{l}110.08 \\
122.18 \\
131.69 \\
121.71 \\
121.67\end{array}$ \\
\hline $\begin{array}{l}699-57-83 B R \\
699-57-83 C \\
699-59-32 \\
699-59-58 \\
699-59-80 B\end{array}$ & $\begin{array}{l}c \\
c\end{array}$ & $\begin{array}{l}54.74 \\
52.65 \\
19.18 \\
29.61 \\
46.89\end{array}$ & $\begin{array}{l}176.45 \\
176.66 \\
129.32 \\
151.72 \\
177.77\end{array}$ & $\begin{array}{l}121.71 \\
124.01 \\
110.14 \\
122.11 \\
130.88\end{array}$ \\
\hline $\begin{array}{l}699-60-32 \\
699-60-57 \\
699-60-59 \\
699-60-60 \\
699-61-37\end{array}$ & $\begin{array}{l}\mathrm{I} \\
\mathrm{D} \\
\mathrm{N}\end{array}$ & $\begin{array}{l}19.53 \\
20.92 \\
32.12 \\
33.96 \\
18.84\end{array}$ & $\begin{array}{l}129.63 \\
143.15 \\
155.45 \\
156.07 \\
135.01\end{array}$ & $\begin{array}{l}110.10 \\
122.22 \\
123.33 \\
122.10 \\
116.17\end{array}$ \\
\hline $\begin{array}{l}699-61-41 \\
699-61-62 \\
699-61-66 \\
699-62-31 \\
699-62-43 A\end{array}$ & $N$ & $\begin{array}{l}10.23 \\
29.53 \\
37.30 \\
22.24 \\
11.19\end{array}$ & $\begin{array}{l}130.73 \\
151.64 \\
159.16 \\
132.32 \\
131.77\end{array}$ & $\begin{array}{l}120.51 \\
122.11 \\
121.86 \\
110.08 \\
120.57\end{array}$ \\
\hline
\end{tabular}


Appendix A: June 1994 Water Level Measurement Data (Sheet 20 of 23)

\begin{tabular}{|c|c|c|c|c|}
\hline We11 & Notes & $\begin{array}{l}\text { Depth to } \\
\text { Water }(\mathrm{m})\end{array}$ & $\begin{array}{r}\text { Elevation, } \\
\text { Adjusted Casing }\end{array}$ & $\begin{array}{l}\text { above ms } 1 \\
\text { Water Level }\end{array}$ \\
\hline $\begin{array}{l}699-63-25 A \\
699-63-51 \\
699-63-55 \\
699-63-58 \\
699-63-90\end{array}$ & & $\begin{array}{r}10.41 \\
7.95 \\
8.36 \\
28.19 \\
33.89\end{array}$ & $\begin{array}{l}120.44 \\
129.40 \\
130.01 \\
149.93 \\
155.37\end{array}$ & $\begin{array}{l}110.03 \\
121.45 \\
121.65 \\
121.74 \\
121.47\end{array}$ \\
\hline $\begin{array}{l}699-63-92 \\
699-64-27 \\
699-64-62 \\
699-65-22 \\
699-65-50\end{array}$ & $\begin{array}{l}\mathrm{C} \\
\mathrm{N}\end{array}$ & $\begin{array}{r}29.89 \\
16.21 \\
30.79 \\
8.65 \\
20.92\end{array}$ & $\begin{array}{l}151.64 \\
126.28 \\
152.48 \\
119.21 \\
142.36\end{array}$ & $\begin{array}{l}121.75 \\
110.07 \\
121.68 \\
110.55 \\
121.44\end{array}$ \\
\hline $\begin{array}{l}699-65-59 A \\
699-65-72 \\
699-65-83 \\
699-65-95 \\
699-66-103\end{array}$ & C & $\begin{array}{l}32.87 \\
43.43 \\
26.90 \\
16.16 \\
19.29\end{array}$ & $\begin{array}{l}154.52 \\
164.68 \\
148.02 \\
137.85 \\
141.13\end{array}$ & $\begin{array}{l}121.65 \\
121.25 \\
121.12 \\
121.69 \\
121.84\end{array}$ \\
\hline $\begin{array}{l}699-66-23 \\
699-66-38 \\
699-66-39 \\
699-66-58 \\
699-66-64\end{array}$ & $\begin{array}{l}N \\
N\end{array}$ & $\begin{array}{r}8.29 \\
10.44 \\
14.38 \\
31.78 \\
32.67\end{array}$ & $\begin{array}{l}118.57 \\
132.97 \\
138.31 \\
153.41 \\
154.20\end{array}$ & $\begin{array}{l}110.28 \\
122.53 \\
123.93 \\
121.64 \\
121.54\end{array}$ \\
\hline $\begin{array}{l}699-66-91 \\
699-67-51 \\
699-67-86 \\
699-67-98 \\
699-68-105\end{array}$ & c & $\begin{array}{l}20.95 \\
38.41 \\
22.91 \\
16.98 \\
16.26\end{array}$ & $\begin{array}{l}142.57 \\
159.90 \\
143.98 \\
138.83 \\
137.72\end{array}$ & $\begin{array}{l}121.62 \\
121.48 \\
121.07 \\
121.84 \\
121.46\end{array}$ \\
\hline $\begin{array}{l}699-69-38 \\
699-69-450 \\
699-70-23 \\
699-70-68 \\
699-71-30\end{array}$ & I & $\begin{array}{r}6.47 \\
27.20 \\
8.46 \\
39.11 \\
9.31\end{array}$ & $\begin{array}{l}129.27 \\
148.49 \\
119.39 \\
160.39 \\
122.13\end{array}$ & $\begin{array}{l}122.80 \\
121.29 \\
110.93 \\
121.28 \\
112.81\end{array}$ \\
\hline $\begin{array}{l}699-71-52 \\
699-71-77 \\
699-72-73 \\
699-72-88 \\
699-72-92\end{array}$ & I & $\begin{array}{l}38.15 \\
23.12 \\
26.35 \\
11.25 \\
15.79\end{array}$ & $\begin{array}{l}159.42 \\
143.95 \\
147.09 \\
133.31 \\
137.84\end{array}$ & $\begin{array}{l}121.27 \\
120.83 \\
120.74 \\
122.06 \\
122.05\end{array}$ \\
\hline
\end{tabular}


Appendix A: June 1994 Water Level Measurement Data

(Sheet 21 of 23)

\begin{tabular}{|c|c|c|c|c|}
\hline We11 & Notes & $\begin{array}{l}\text { Depth to } \\
\text { Water (m) }\end{array}$ & $\begin{array}{l}\text { Elevation, } \\
\text { Adjusted Casing }\end{array}$ & $\begin{array}{l}\text { ve ms } 1 \\
\text { Water Level }\end{array}$ \\
\hline $\begin{array}{l}699-72-98 \\
699-73-61 \\
699-74-44 \\
699-74-48 \\
699-77-36\end{array}$ & D & $\begin{array}{l}15.21 \\
40.81 \\
14.92 \\
27.72 \\
11.16\end{array}$ & $\begin{array}{l}138.44 \\
162.01 \\
135.69 \\
148.49 \\
125.66\end{array}$ & $\begin{array}{l}123.22 \\
121.20 \\
120.77 \\
120.77 \\
114.50\end{array}$ \\
\hline $\begin{array}{l}699-77-54 \\
699-78-62 \\
699-8-17 \\
699-8-25 \\
699-8-32\end{array}$ & & $\begin{array}{l}25.79 \\
23.19 \\
38.16 \\
33.80 \\
47.35\end{array}$ & $\begin{array}{l}146.48 \\
143.22 \\
159.24 \\
155.23 \\
168.98\end{array}$ & $\begin{array}{l}120.69 \\
120.03 \\
121.08 \\
121.44 \\
121.63\end{array}$ \\
\hline $\begin{array}{l}699-80-435 \\
699-81-38 \\
699-81-58 \\
699-82-45 A \\
699-83-36\end{array}$ & $D$ & $\begin{array}{r}7.77 \\
8.43 \\
14.38 \\
7.82 \\
12.58\end{array}$ & $\begin{array}{l}125.74 \\
123.89 \\
133.97 \\
126.10 \\
127.60\end{array}$ & $\begin{array}{l}117.97 \\
115.46 \\
119.60 \\
118.29 \\
115.02\end{array}$ \\
\hline $\begin{array}{l}699-83-47 \\
699-84-35 A \\
699-86-42 \\
699-87-42 A \\
699-87-55\end{array}$ & $c$ & $\begin{array}{r}14.36 \\
2.16 \\
7.82 \\
10.05 \\
22.15\end{array}$ & $\begin{array}{l}132.67 \\
121.94 \\
124.94 \\
126.96 \\
139.79\end{array}$ & $\begin{array}{l}118.31 \\
119.78 \\
117.13 \\
116.91 \\
117.64\end{array}$ \\
\hline $\begin{array}{l}699-88-41 \\
699-89-35 \\
699-9-E 2 \\
699-90-34 \\
699-90-37 B\end{array}$ & $\begin{array}{l}D \\
D\end{array}$ & $\begin{array}{r}10.41 \\
7.68 \\
13.99 \\
6.04 \\
15.22\end{array}$ & $\begin{array}{l}126.81 \\
121.15 \\
127.43 \\
119.60 \\
128.91\end{array}$ & $\begin{array}{l}116.40 \\
113.46 \\
113.45 \\
113.56 \\
113.69\end{array}$ \\
\hline $\begin{array}{l}699-90-45 \\
699-91-46 A \\
699-93-48 A \\
699-96-43 \\
699-96-49\end{array}$ & $\begin{array}{l}D \\
D\end{array}$ & $\begin{array}{l}11.54 \\
10.22 \\
16.85 \\
13.13 \\
10.56\end{array}$ & $\begin{array}{l}128.49 \\
127.12 \\
133.44 \\
128.58 \\
127.78\end{array}$ & $\begin{array}{l}116.95 \\
116.90 \\
116.59 \\
115.45 \\
117.22\end{array}$ \\
\hline $\begin{array}{l}699-97-43 \\
699-97-51 A \\
699-98-49 A \\
699-S 12-29 \\
699-S 12-3\end{array}$ & D & $\begin{array}{r}13.24 \\
6.32 \\
6.27 \\
25.49 \\
16.65\end{array}$ & $\begin{array}{l}128.58 \\
122.61 \\
122.47 \\
148.64 \\
132.75\end{array}$ & $\begin{array}{l}115.34 \\
116.30 \\
116.20 \\
123.16 \\
116.10\end{array}$ \\
\hline
\end{tabular}


Appendix A: June 1994 Water Leve1 Measurement Data (Sheet 22 of 23)

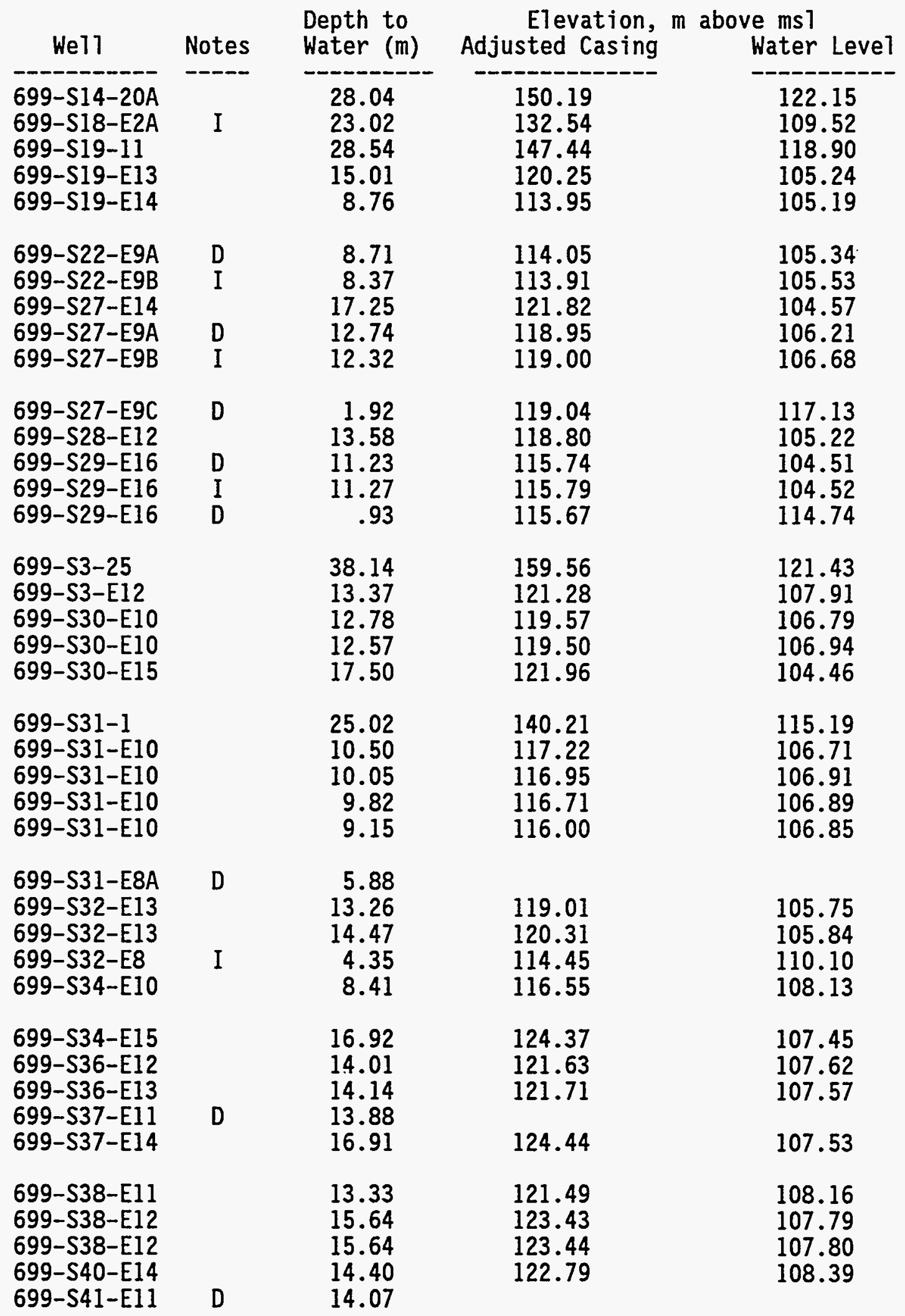


Appendix A: June 1994 Water Level Measurement Data (Sheet 23 of 23)

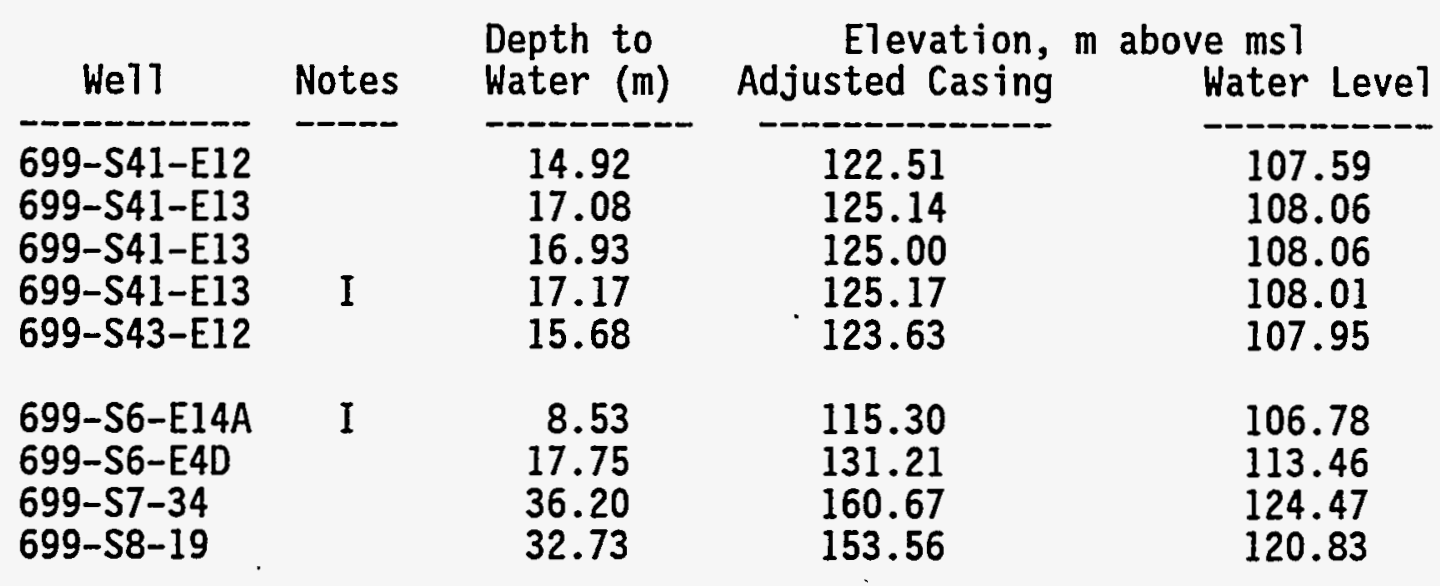

Notes: $\quad C=$ Well in confined aquifer, not included in model

$D=$ Insufficient construction data, not included in model

$I$ = Inadequate well construction, not included in model

$N=$ Well included in model but not shown on map

$Q=$ Questionable data, not included in model 
WHC-EP-0394-9

This page intentionally left blank. 


\section{DISTRIBUTION}

Number of Copies

\section{offsite}

1 U.S. Geological Survey

1201 Pacific Ave.

Suite 600

Tacoma, Wa. 98402

B. D. Drost

Onsite

$4 \quad$ U.S. Department of Energy

Richland, Operations office

M. J. Furman

A. J. Lassila

R3-80

S7-52

K. M. Thompson

R. D. Hildebrand

2 Washington State Department of Ecology

S. Leja

$\mathrm{N} 1-08$

M. A. Selby

N1-08

2 U.S. Environmental Protection Agency

D. R. Sherwood (2)

B5-01

12 Pacific Northwest Laboratory

M. P. Bergeron

K6-77

$\mathrm{K} 6-96$

M. A. Chamness

K6-84

K6-77

M. D. Freshley

K6-84

S. M. Goodwin

K6-96

E. L. Hilty

G. V. Last

S. P. Luttrell

D. R. Newcomer

R. M. Smith

F. A. Spane

$\mathrm{K} 6-84$

K6-96

K6-96

K6-96

$\mathrm{K} 7-96$

W. D. Hebber

K6-96 
WHC-EP-0394-9

DISTRIBUTION (cont.)

38 Westinghouse Hanford Company

M. R. Adams

H6-30

D. J. Alexander

D. B. Barnett

J. A. Caggiano

L. B. Collard

J. D. Davis

L. P. Diediker

J. J. Dorian

G. L. Dunford

J. W. Fassett

M. A. Frank

M. G. Gardner

M. J. Hartman (5)

F. N. Hodges

D. G. Horton

V. G. Johnson

H. A. Jordan

H6-06

H6-06

H6-06

H6-01

H6-01

T1-30

H6-30

R1-51

H6-06

H6-03

N3-06

H6-06

H6-06

H6-06

H6-06

H6-06

J. W. Lindberg

H6-06

A. H. Lu

R. B. Mercer

HO-36

H6-06

R. D. Miller

D. J. Moak

H6-06

N3-05

S. E. Myers

S. M. Narbutovskih

N3-06

H6-06

R. S. Pavlina

S. M. Price

S. P. Reidel

K. D. Reynolds

A. L. Schatz

J. S. Schmid

J. A. Serkowski

T7-39

H6-23

H6-06

H6-06

N3-05

H6-06

H6-06

M. D. Sweeney

H6-06

H6-32

H6-06

L8-04

L8-07

H6-08

EPIC (2)

Public Reading Room

Al-65

$9 \quad \mathrm{CH} 2 \mathrm{M} \mathrm{Hil1}$

J. V. Borghese

R. S. Edrington

R. L. Jackson

K. A. Lindsey

H. J. McMahon

H4-9O

H6-03

H4-90

H6-02

H4-90

R. E. Peterson

H6-05 


\section{CH2M Hill (cont.)}
R. F. RaidT
H4-89
D. K. Tyler
H4-89
P. J. VaTcich
H4-90

6 Bechtel Hanford Incorporated
M. A. Buckmaster
K. R. Fecht
B. H. Ford
A. J. Knepp
W. H. Price
S. J. Trent

H6-01

$\mathrm{H} 4-85$

H4-85

H4-85

H4-86

H4-85

8 IT Corporation, Inc.
B. E. Innis
D. B. Erb
G. L. Kasza
V. J. Rohay
K. R. Simpson
L. C. Swanson
D. C. Weekes
C. D. Wittreich

H6-03

H6-0I

H6-04

H6-02

H6-06

H6-03

H4-82

H6-02 
WHC-EP-0394-9

This page intentionally left blank.

Distr-4 